ESAIM: M2AN 55 (2021) 533-560

https://doi.org/10.1051/m2an/2020085
ESAIM: Mathematical Modelling and Numerical Analysis

www.esaim-m2an.org

\title{
A VIRTUAL ELEMENT METHOD FOR THE VON KÁRMÁN EQUATIONS
}

\author{
Carlo Lovadina ${ }^{1}$, David Mora $^{2,3, *}$ and Iván Velásquez ${ }^{4}$
}

\begin{abstract}
In this article we propose and analyze a Virtual Element Method (VEM) to approximate the isolated solutions of the von Kármán equations, which describe the deformation of very thin elastic plates. We consider a variational formulation in terms of two variables: the transverse displacement of the plate and the Airy stress function. The VEM scheme is conforming in $H^{2}$ for both variables and has the advantages of supporting general polygonal meshes and is simple in terms of coding aspects. We prove that the discrete problem is well posed for $h$ small enough and optimal error estimates are obtained. Finally, numerical experiments are reported illustrating the behavior of the virtual scheme on different families of meshes.
\end{abstract}

Mathematics Subject Classification. 65N30, 65N12, 74K20, 74S05, 65N15.

Received September 6, 2019. Accepted December 14, 2020.

\section{INTRODUCTION}

The von Kármán equations is a fourth order system of nonlinear partial differential equations that model the deformation of very thin plates. This system consists of two unknowns, which describe the transverse displacement or deflection of the plate from its flat unstressed position and the Airy stress function governing the in-plane stress resultants (see [31]). This model has attracted great interest in the scientific community since it is frequently encountered in several engineering applications such as bridge deck analysis (see [37, 49]).

Results on existence of solutions of the von Kármán system have been stated in [31,33]. In general the problem does not have a unique solution. However, sufficient conditions that guarantee uniqueness of isolated solutions are established in [23]. Due to the importance of this problem, several finite element methods have been developed to approximate the isolated solutions of a von Kármán plate. For instance, a general technique based on any conforming discretization was introduced in [23], where convergence and optimal error bounds in the energy norm are presented considering the standard formulation in $H^{2}$. Then, in [40] a conforming finite element method was analyzed to approximate the isolated solutions of the von Kármán problem, using Bogner-Fox-Schmit elements, and they also obtained error estimates in $H^{1}$ and $L^{2}$ norms using a duality argument. On the other hand, to avoid $C^{1}$ finite elements, nonconforming discretizations based on Morley finite element methods were proposed in $[39,41]$. In these works, a priori error estimates for displacement and Airy stress functions have been established. Lately, a $C^{0}$ interior penalty method has been introduced in [20]. The method uses quadratic Lagrange elements

Keywords and phrases. Virtual element method, von Kármán equations, error estimates, polygonal meshes.

1 Dipartimento di Matematica, Università di Milano, Via Saldini 50, 20133 Milano, Italy.

2 GIMNAP, Departamento de Matemática, Universidad del Bío-Bío, Concepción, Chile.

$3 \mathrm{CI}^{2} \mathrm{MA}$, Universidad de Concepción, Concepción, Chile.

4 Departamento de Ciencias Básicas, Universidad del Sinú-Elías Bechara Zainúm, Montería, Colombia.

* Corresponding author: dmora@ubiobio.cl 
to approximate both the transverse displacement and the Airy stress function. Optimal order error estimates are derived. More recently, a discontinuous Galerkin method has been developed in [28]. The authors prove a priori and a posteriori error estimates for the isolated solution of von Kármán equations.

It is well known that conforming finite element spaces of $H^{2}$ are of complex implementation and contain high order polynomials (see [32]), for instance, Argyris and Bell finite elements (21 and 18 degrees of freedom per triangle, respectively) or Bogner-Fox-Schmit finite elements (16 degrees of freedom in a rectangle), respectively. In this paper, we will propose a $C^{1}$ VEM to approximate the isolated solutions of the von Kármán problem which can be applied to general polygonal meshes (made by possibly non-convex elements). The method will make use of a very simple set of degrees of freedom. In particular, the total computational cost of the proposed VEM method will be $6 N_{v}$, where $N_{v}$ denotes the number of internal vertices of the polygonal mesh to approximate both the transverse displacement and the Airy stress function.

The VEM was introduced for the first time in [8], as a generalization of the finite element methods by considering polygonal or polyhedral meshes. One of its main characteristics is the possibility to construct and implement in an easy way discrete subspaces of $C^{\alpha}, \alpha \in \mathbb{N}$. In recent years, the Virtual Elements Method has been a focus of great interest in the scientific community. Several virtual element methods based on conforming and non-conforming schemes have been developed to solve a wide variety of problems in Solid and Fluid Mechanics, for example [4-6,9,11,12,14,19,25,27,30,42,46,47]. Moreover, the VEM for thin structures has been developed in $[16,24,29,30,44,45]$, whereas VEM for nonlinear problems have been introduced in $[3,15,26,35,36,50]$

In this paper, we analyze a conforming $C^{1}$ Virtual Element Method to approximate the isolated solutions of the von Kármán equations. We consider a variational formulation in terms of the transverse displacement and the Airy stress function, which contains bilinear and trilinear forms. After introducing the local and global virtual space $[5,24,30]$, we write the discrete problem by constructing discrete version of the bilinear and trilinear forms considering different projectors (polynomial functions) which are computable using only the information of the degrees of freedom of the discrete virtual space. For the analysis, we will adapt some ideas presented in [23] to deal with the variational crimes in the forms and in the right hand side. More precisely, in order to prove that the discrete scheme is well posed, we introduce an ad-hoc operator $T_{h}$ which relates each solution of the discrete problem as a fixed point of this operator (and reciprocally). To prove existence and uniqueness the classical Banach fixed point theorem is employed and some assumptions on the mesh are considered. In particular, for $h$ small enough, we establish that the operator $T$ has a unique fixed point in a proper set which is the unique solution of the discrete problem. Optimal order of convergence in $H^{2}$-norm is established in this work for both unknowns.

The outline of this work is organized as follows. In Section 2 the physical model problem is described. An auxiliary variable that depends of the horizontal load forces applied to the plate is introduced, which allows us to rewrite an equivalent nonlinear system of partial differential equations. Hence, a variational formulation is obtained from this system. In Section 3, we introduce a conforming virtual element discretization and some auxiliary local results are proved. Then, in Section 4, fixed-point arguments are employed to establish that our discrete scheme is well posed. In addition, optimal convergence rate is obtained in this section. Finally, in Section 5, we report some numerical tests that confirm the theoretical analysis developed.

In this article, we will employ standard notations for Sobolev spaces, norms and seminorms. In addition, we will denote with $c$ and $C$, with or without subscripts, tildes or hats, a generic constants independent of the mesh parameter $h$, which may take different values in different occurrences. In addition, let $X, Y$ be Hilbert spaces. If $\Pi: X \rightarrow Y$ is a linear and bounded operator, we will denote by $\Pi: X \times X \rightarrow Y \times Y$ the operator defined by $\Pi(x, \tilde{x}):=(\Pi x, \Pi \tilde{x}), \forall(x, \tilde{x}) \in X \times X$.

\section{The CONTINUOUS PROBLEM}

Let $\Omega \subset \mathbb{R}^{2}$ be a polygonal bounded domain with boundary $\Gamma:=\partial \Omega$. The von Kármán system can be read as follows (see e.g. [31,33]): Given a load force $f \in L^{2}(\Omega)$ and lateral load forces $\left(\varphi_{0}, \varphi_{1}\right) \in H^{5 / 2}(\Gamma) \times H^{3 / 2}(\Gamma)$ 
(see Fig. 1), find $u$ and $\phi$ such that

$$
\begin{aligned}
\Delta^{2} u & =[\phi, u]+f & & \text { in } \Omega, \\
\Delta^{2} \phi & =-\frac{1}{2}[u, u] & & \text { in } \Omega, \\
u=\partial_{\nu} u & =0 & & \text { on } \Gamma, \\
\phi & =\varphi_{0} & & \text { on } \Gamma, \\
\partial_{\nu} \phi & =\varphi_{1} & & \text { on } \Gamma,
\end{aligned}
$$

where $[\phi, u]$ is defined by

$$
[\phi, u]:=\partial_{11} \phi \partial_{22} u+\partial_{22} \phi \partial_{11} u-2 \partial_{12} \phi \partial_{12} u,
$$

and $\nu:=\left(\nu_{1}(x, y), \nu_{2}(x, y)\right)$ denotes the outer unit normal vector to $\Gamma$, for all $(x, y) \in \Gamma$.

In the system (2.1), $u$ and $\phi$ represent the transverse displacement and the boundary stresses of the plate, respectively. This model is also known as the canonical von Kármán equations. This is a non-linear system of fourth-order partial differential equations established by T. von Kármán in 1910 (see [48]). The existence of solutions of this problem has been proved in [31,33]. Moreover, it can be seen in Section 2.2 of [33] that introducing $\theta_{0} \in H_{0}^{2}(\Omega)$ as the unique solution of the following problem: find $\theta_{0} \in H_{0}^{2}(\Omega)$ such that

$$
\Delta^{2} \theta_{0}=0 \text { in } \Omega, \quad \theta_{0}=\varphi_{0}, \quad \partial_{\nu} \theta_{0}=\varphi_{1} \quad \text { on } \Gamma,
$$

we have that system (2.1) can be written equivalently as the following problem with homogeneous clamped boundary conditions: find $(u, \psi) \in\left[H_{0}^{2}(\Omega)\right]^{2}$ such that

$$
\begin{aligned}
\Delta^{2} u & =[\psi, u]+\left[\theta_{0}, u\right]+f & & \text { in } \Omega, \\
\Delta^{2} \psi & =-\frac{1}{2}[u, u] & & \text { in } \Omega, \\
u=\partial_{\nu} u & =\psi=\partial_{\nu} \psi=0 & & \text { on } \Gamma,
\end{aligned}
$$

where $\psi=\phi-\theta_{0}$.

Moreover, in this paper we consider the physical case corresponding to a uniform lateral loading along the plate boundary, most relevant in buckling analysis. Accordingly (see [33], Sect. 2.3 or [31], Sect. 5.9 for further details), in equation (2.3) we set:

$$
\varphi_{0}:=-\frac{\lambda}{2}\left(x^{2}+y^{2}\right) \quad \text { on } \Gamma, \quad \varphi_{1}:=-\frac{\lambda}{2} \partial_{\nu}\left(x^{2}+y^{2}\right) \quad \text { on } \Gamma,
$$

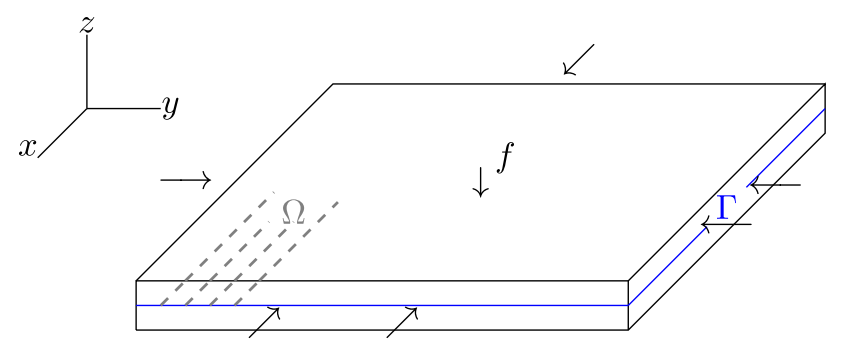

$\varphi_{0}, \varphi_{1}$

Figure 1. Plate subject to transversal and lateral forces $f \in L^{2}(\Omega)$ and $\left(\varphi_{0}, \varphi_{1}\right) \in H^{5 / 2}(\Gamma) \times$ $H^{3 / 2}(\Gamma)$, respectively. 
where $\lambda$ is a real number called bifurcation parameter that measures the intensity of the horizontal forces; then we obtain that $\theta_{0}(x, y)=-\frac{\lambda}{2}\left(x^{2}+y^{2}\right)$ in $\Omega$ solves problem $(2.3)$.

In addition, from the definition of $[\cdot, \cdot]$, we obtain that

$$
\left[\theta_{0}, u\right]=-\lambda \Delta u \quad \text { in } \Omega .
$$

As a consequence of the previous discussion, from now on, our aim is to solve the following set of equations: given $(f, \lambda) \in L^{2}(\Omega) \times \mathbb{R}$, find $(u, \psi) \in\left[H_{0}^{2}(\Omega)\right]^{2}$ such that

$$
\begin{aligned}
\Delta^{2} u & =[\psi, u]-\lambda \Delta u+f & & \text { in } \Omega, \\
\Delta^{2} \psi & =-\frac{1}{2}[u, u] & & \text { in } \Omega, \\
u=\partial_{\nu} u & =\psi=\partial_{\nu} \psi=0 & & \text { on } \Gamma .
\end{aligned}
$$

Remark 2.1. The von Kármán model (2.4) does not have a unique solution: For instance, when $f=0$ and $\lambda>\lambda^{\star}$, where $\lambda^{\star}$ is the lowest positive real number that satisfies

$$
\Delta^{2} u=-\lambda \Delta u \quad \text { in } \Omega \quad \text { and } \quad u=\partial_{\nu} u=0 \text { on } \Gamma,
$$

the problem (2.4) has at least three solutions: $(u, \psi)=0$ (trivial solution) and two non-trivial solutions with identical $\psi$ and transverse displacement with opposite signs $( \pm u, \psi)$ (see [31], Thm. 5.9-2). On the other hand, if $f$ and $\lambda$ are small enough then the system (2.4) has a unique solution.

Now, testing system (2.4) with functions in $H_{0}^{2}(\Omega)$, we arrive at the following weak formulation of the problem: given $(f, \lambda) \in L^{2}(\Omega) \times \mathbb{R}$, find $(u, \psi) \in\left[H_{0}^{2}(\Omega)\right]^{2}$ such that

$$
\begin{aligned}
a^{\Delta}(u, v)+\lambda a^{\nabla}(u, v)+b(u, \psi, v)+b(\psi, u, v) & =F(v) & & \forall v \in H_{0}^{2}(\Omega), \\
a^{\Delta}(\psi, \varphi)-b(u ; u, \varphi) & =0 & & \forall \varphi \in H_{0}^{2}(\Omega),
\end{aligned}
$$

where $a^{\Delta}, a^{\nabla}: H_{0}^{2}(\Omega) \times H_{0}^{2}(\Omega) \rightarrow \mathbb{R}$ are bilinear forms, $b: H_{0}^{2}(\Omega) \times H_{0}^{2}(\Omega) \times H_{0}^{2}(\Omega) \rightarrow \mathbb{R}$ is a trilinear form and $F: H_{0}^{2}(\Omega) \rightarrow \mathbb{R}$ is a linear functional, all of them defined as follows:

$$
a^{\Delta}(u, v):= \begin{cases}\int_{\Omega} \Delta u \Delta v & \forall u, v \in H_{0}^{2}(\Omega) \\ \text { or } & \\ \int_{\Omega} D^{2} u: D^{2} v & \forall u, v \in H_{0}^{2}(\Omega),\end{cases}
$$

where $D^{2} u:=\left(\partial_{i j} u\right)_{1 \leq i, j \leq 2}$ denotes the Hessian matrix of $u$ and ":" denotes the usual scalar product of $2 \times 2$ matrices.

$$
\begin{aligned}
a^{\nabla}(u, v) & :=-\int_{\Omega} \nabla u \cdot \nabla v & & \forall u, v \in H_{0}^{2}(\Omega), \\
b(w ; u, v) & :=-\frac{1}{2} \int_{\Omega}[w, u] v & & \forall w, u, v \in H_{0}^{2}(\Omega), \\
F(v) & :=\int_{\Omega} f v & & \forall v \in H_{0}^{2}(\Omega) .
\end{aligned}
$$

Remark 2.2. We have proposed two options to write the bilinear form associated to the bilaplacian operator. We observe that both bilinear forms are equivalent for functions in $H_{0}^{2}(\Omega)$. We will write the discrete method considering both options for the sake of completeness. In particular, we will employ the bilinear form $a^{\Delta}(u, v):=$ $\int_{\Omega} D^{2} u: D^{2} v$ to construct the projector $\Pi_{K}^{2, D}(c f$. (3.1a) and (3.1b)) which will be used to write the discrete schemes with the two options in (2.8). 
On the other hand, we endow the space $\mathbb{H}:=\left[H_{0}^{2}(\Omega)\right]^{2}$ with the corresponding product norm.

We rewrite (2.6) and (2.7), in the following equivalent form: given $(f, \lambda) \in L^{2}(\Omega) \times \mathbb{R}$, find $\mathbf{u}:=(u, \psi) \in \mathbb{H}$ such that

$$
A^{\Delta}(\mathbf{u}, \mathbf{v})+\lambda A^{\nabla}(\mathbf{u}, \mathbf{v})+B(\mathbf{u} ; \mathbf{u}, \mathbf{v})=F(\mathbf{v}) \quad \forall \mathbf{v}:=(v, \varphi) \in \mathbb{H},
$$

where, $A^{\Delta}, A^{\nabla}, B, F$ are defined as follows:

$$
\begin{aligned}
A^{\Delta}(\mathbf{u}, \mathbf{v}) & :=a^{\Delta}(u, v)+a^{\Delta}(\psi, \varphi) & & \forall \mathbf{u}, \mathbf{v} \in \mathbb{H}, \\
A^{\nabla}(\mathbf{u}, \mathbf{v}) & :=a^{\nabla}(u, v) & & \forall \mathbf{u}, \mathbf{v} \in \mathbb{H}, \\
B(\mathbf{w} ; \mathbf{u}, \mathbf{v}) & :=b(w ; \psi, v)+b(\xi ; u, v)-b(w ; u, \varphi) & & \forall \mathbf{w}, \mathbf{u}, \mathbf{v} \in \mathbb{H}, \\
F(\mathbf{v}) & :=F(v) & & \forall \mathbf{v} \in \mathbb{H},
\end{aligned}
$$

with $\mathbf{w}:=(w, \xi), \mathbf{u}:=(u, \psi), \mathbf{v}:=(v, \varphi)$.

It is easy to see that the bilinear forms $a^{\Delta}(\cdot, \cdot)$ and $a^{\nabla}(\cdot, \cdot)$ are bounded and symmetric, the former is also positive definite on $H_{0}^{2}(\Omega) \times H_{0}^{2}(\Omega)$ and $F$ is bounded. Moreover, from Lemma 2.2-2 in [33] we have that the trilinear form $b(\cdot ; \cdot, \cdot)$ is bounded and symmetric independent of the arguments. Therefore, we have the following result.

Lemma 2.1. The forms defined in (2.13)-(2.16) satisfy the following properties:

$$
\begin{aligned}
\left|A^{\Delta}(\mathbf{u}, \mathbf{v})\right| & \leq\|\mathbf{u}\|_{2, \Omega}\|\mathbf{v}\|_{2, \Omega} \quad \text { and } \quad\left|A^{\nabla}(\mathbf{u}, \mathbf{v})\right| \leq|\lambda|\|\mathbf{u}\|_{2, \Omega}\|\mathbf{v}\|_{2, \Omega} & & \forall \mathbf{u}, \mathbf{v} \in \mathbb{H}, \\
|F(\mathbf{v})| & \leq\|f\|_{0, \Omega}\|\mathbf{v}\|_{2, \Omega} & & \forall \mathbf{u}, \mathbf{v} \in \mathbb{H}, \\
|B(\mathbf{w} ; \mathbf{u}, \mathbf{v})| & \leq C\|\mathbf{w}\|_{2, \Omega}\|\mathbf{u}\|_{2, \Omega}\|\mathbf{v}\|_{2, \Omega} & & \forall \mathbf{w}, \mathbf{u}, \mathbf{v} \in \mathbb{H}, \\
B(\mathbf{w} ; \mathbf{u}, \mathbf{v}) & =B(\mathbf{u} ; \mathbf{w}, \mathbf{v}) & & \forall \mathbf{w}, \mathbf{u}, \mathbf{v} \in \mathbb{H} .
\end{aligned}
$$

Now, from Theorem 5.8-3(b) of [31] (see also [38]) we have that the variational formulation (2.12) has at least one solution. Moreover, we present the following additional regularity result for the solution of the von Kármán problem (2.12), which has been proved in Theorem 2.4 of [20].

Theorem 2.1. Let $\mathbf{u}$ be a solution of von Kármán problem (2.12). Then, there exist $s \in(1 / 2,1]$ and $C>0$ such that $\mathbf{u} \in\left[H^{2+s}(\Omega)\right]^{2}$ and

$$
\|\mathbf{u}\|_{2+s, \Omega} \leq C\|f\|_{0, \Omega},
$$

where, the constant $s \in(1 / 2,1]$ is the Sobolev regularity for the biharmonic equation with the right hand side in $H^{-1}(\Omega)$ and homogeneous Dirichlet boundary conditions (see e.g. [18] and [20], Lem. 1.1).

Now, we will introduce some definitions that will be used to establish an existence result of the isolated solutions of the problem (2.12). Given $\mathbf{u} \in \mathbb{H}$ we introduce the following global form.

$$
A_{\mathbf{u}}(\mathbf{w}, \mathbf{v}):=A^{\Delta}(\mathbf{w}, \mathbf{v})+\lambda A^{\nabla}(\mathbf{w}, \mathbf{v})+2 B(\mathbf{u} ; \mathbf{w}, \mathbf{v}) \quad \forall \mathbf{w}, \mathbf{v} \in \mathbb{H} .
$$

Definition 2.2. (see [23]) A solution $\mathbf{u}$ of the system (2.12) is said to be isolated if and only if the linearized problem: given $\mathbf{g} \in\left[L^{2}(\Omega)\right]^{2}$, find $\mathbf{w} \in \mathbb{H}$ such that

$$
A_{\mathbf{u}}(\mathbf{w}, \mathbf{v})=\int_{\Omega} \mathbf{g} \cdot \mathbf{v} \quad \forall \mathbf{v} \in \mathbb{H},
$$

has a unique solution and satisfies the following a priori estimates

$$
\|\mathbf{w}\|_{2, \Omega} \leq C\|\mathbf{g}\|_{0, \Omega} \quad \text { and } \quad\|\mathbf{w}\|_{2+s, \Omega} \leq C\|\mathbf{g}\|_{0, \Omega},
$$

where, the constant $s \in(1 / 2,1]$ is the Sobolev regularity for the biharmonic problem with the right hand side in $H^{-1}(\Omega)$ and homogeneous Dirichlet boundary conditions (see e.g. [18] and [20], Lem. 1.1). 
Now, the following result gives a sufficient condition to obtain an isolated solution of the system (2.12) (see for instance [20], Rem. 3.1, or [40], Rem. 2.1-[40], Thm. 2.3).

Theorem 2.3. If $(f, \lambda) \in L^{2}(\Omega) \times \mathbb{R}$ are small enough, then the von Kármán system (2.12) has a unique solution and it is isolated.

We finish this section with the following theorem which will be used to approximate the isolated solution of the von Kármán problem. The result is based on the well-known Banach-Nečas-Babuška Theorem (see [34], Thm. 2.6).

Theorem 2.4. Assume that the bilinear form $A_{\mathbf{u}}(\cdot, \cdot)$ (cf. (2.17)) is non singular on $\mathbb{H} \times \mathbb{H}$, this means (see e.g. [23], Lem. 1) that there exist two positive constants $c_{1}$ and $c_{2}$ such that

$$
\sup _{\| \mathbf{v} \in \mathbb{H}} A_{\|_{2, \Omega}=1} A_{\mathbf{u}}(\mathbf{w}, \mathbf{v}) \geq c_{1}\|\mathbf{w}\|_{2, \Omega} \forall \mathbf{w} \in \mathbb{H}, \quad \text { and } \quad \sup _{\substack{\mathbf{w} \in \mathbb{H} \\\|\mathbf{w}\|_{2, \Omega}=1}} A_{\mathbf{u}}(\mathbf{w}, \mathbf{v}) \geq c_{2}\|\mathbf{v}\|_{2, \Omega} \forall \mathbf{v} \in \mathbb{H} \text {. }
$$

Then, there exists a positive constant $\delta$, which depends on $c_{1}$ and $c_{2}$, such that $A_{\tilde{\mathbf{u}}}(\cdot, \cdot)$ is non singular on $\mathbb{H} \times \mathbb{H}$, for all $\tilde{\mathbf{u}}$ that satisfies

$$
\|\mathbf{u}-\tilde{\mathbf{u}}\|_{2, \Omega} \leq \delta
$$

Proof. The proof can be obtained repeating the arguments in Lemma 1 of [23].

\section{Discrete PRoblem}

In this section, we will introduce a $C^{1}$-VEM discretization to approximate the isolated solutions of a von Kármán plate ( $c f$. Thm. 2.3).

We begin with the mesh construction and the assumptions considered to introduce the discrete virtual element spaces (see e.g. $[2,5,8]$ ). Let $\left\{\mathcal{T}_{h}\right\}_{h}$ be a sequence of decompositions of $\Omega$ into general polygonal elements $K$. We will denote by $h_{K}$ the diameter of the element $K$ and by $h$ the maximum of the diameters of all the elements of the mesh, i.e., $h:=\max _{K \in \mathcal{T}_{h}} h_{K}$. In addition, we denote by $N_{K}$ the number of vertices of $K$, by $e$ a generic edge of $\left\{\mathcal{T}_{h}\right\}_{h}$ and for all $e \in \partial K$, we define a unit normal vector $\nu_{K}^{e}$ that points outside of $K$.

Moreover, we will make the following assumptions: there exists a positive real number $C_{\mathcal{T}}$ such that, for every $h$ and every $K \in \mathcal{T}_{h}$ :

A1: $K \in \mathcal{T}_{h}$ is star-shaped with respect to every point of a ball of radius $C_{\mathcal{T}} h_{K}$;

A2: the ratio between the shortest edge and the diameter $h_{K}$ of $K$ is larger than $C_{\mathcal{T}}$.

The hypotheses A1 and A2 though not too restrictive in several practical cases, can be further relaxed, as established in [13].

In order to write the method, we first define the following finite dimensional space (see [5]).

$$
\begin{array}{r}
\widetilde{H}_{h}^{K}:=\left\{v_{h} \in H^{2}(K): \Delta^{2} v_{h} \in \mathbb{P}_{2}(K),\left.v_{h}\right|_{\partial K} \in C^{0}(\partial K),\left.v_{h}\right|_{e} \in \mathbb{P}_{3}(e) \forall e \in \partial K,\right. \\
\left.\left.\nabla v_{h}\right|_{\partial K} \in C^{0}(\partial K)^{2},\left.\partial_{\nu_{K}^{e}} v_{h}\right|_{e} \in \mathbb{P}_{1}(e) \forall e \in \partial K\right\} .
\end{array}
$$

It is easy to see that $\mathbb{P}_{2}(K) \subseteq \widetilde{H}_{h}^{K}$.

Next, we introduce two sets of linear operators from $\widetilde{H}_{h}^{K}$ into $\mathbb{R}$. For all $v_{h} \in \widetilde{H}_{h}^{K}$, they are defined as follows:

$\mathbf{D}_{1}$ : evaluation of $v_{h}$ at the $N_{K}$ vertices of $K$;

$\mathbf{D}_{\mathbf{2}}$ : evaluation of $\nabla v_{h}$ at the $N_{K}$ vertices of $K$. 
Now, we will introduce some preliminary definitions in order to construct the discrete scheme. Let $a_{K}^{D}$ : $H^{2}(K) \times H^{2}(K) \rightarrow \mathbb{R}$ be defined as follows:

$$
a_{K}^{D}(u, v):=\int_{K} D^{2} u: D^{2} v, \quad u, v \in H^{2}(K)
$$

We build the projection operator $\Pi_{K}^{2, D}: \tilde{H}_{h}^{K} \rightarrow \mathbb{P}_{2}(K) \subseteq \tilde{H}_{h}^{K}$, defined by the unique solution of the following local problem:

$$
\begin{aligned}
a_{K}^{D}\left(\Pi_{K}^{2, D} v_{h}, q\right) & =a_{K}^{D}\left(v_{h}, q\right) \quad \forall q \in \mathbb{P}_{2}(K), \\
\widehat{\Pi_{K}^{2, D} v_{h}} & =\widehat{v_{h}}, \quad \widehat{\nabla \Pi_{K}^{2, D} v_{h}}=\widehat{\nabla v_{h}},
\end{aligned}
$$

where $\widehat{v_{h}}$ is defined as follows:

$$
\widehat{v_{h}}:=\frac{1}{N_{K}} \sum_{i=1}^{N_{K}} v_{h}\left(\mathrm{v}_{i}\right) \quad \forall v_{h} \in C^{0}(\partial K)
$$

and $\mathrm{v}_{i}, 1 \leq i \leq N_{K}$, are the vertices of $K$.

We note that the bilinear form $a_{K}^{D}(\cdot, \cdot)$ has a non-trivial kernel, given by $\mathbb{P}_{1}(K)$. Thus, the role of condition (3.1b) is to choose an element of the kernel of the operator. In order to show that the projector $\Pi_{K}^{2, D}$ is computable from the output values of the sets $\mathbf{D}_{\mathbf{1}}$ and $\mathbf{D}_{\mathbf{2}}$, we integrate twice by parts on the right hand side of (3.1a) to get that

$$
a_{K}^{D}\left(\Pi_{K}^{2, D} v_{h}, q\right)=\int_{\partial K}\left(D^{2} q \nu_{K}^{e}\right) \cdot \nabla v_{h} \quad \forall q \in \mathbb{P}_{2}(K) .
$$

Hence, we have that the operator $\Pi_{K}^{2, D}$ is well defined on $\widetilde{H}_{h}^{K}$ and is computable from the output values of the sets $\mathbf{D}_{\mathbf{1}}$ and $\mathbf{D}_{\mathbf{2}}$ (see $[5,24]$ ).

Next, we introduce our local virtual space:

$$
H_{h}^{K}:=\left\{v_{h} \in \widetilde{H}_{h}^{K}: \int_{K}\left(v_{h}-\Pi_{K}^{2, D} v_{h}\right) q=0, \quad \forall q \in \mathbb{P}_{2}(K)\right\} .
$$

Note that $H_{h}^{K} \subseteq \widetilde{H}_{h}^{K}$. This allows us to obtain the well definition of $\Pi_{K}^{2, D}$ on $H_{h}^{K}$, and therefore to prove that $\Pi_{K}^{2, D}$ is computable from the output values of operators $\mathbf{D}_{\mathbf{1}}$ and $\mathbf{D}_{\mathbf{2}}$. Moreover, it is easy to check that $\mathbb{P}_{2}(K) \subseteq H_{h}^{K}$. This will guarantee the good approximation properties for the virtual space.

On the other hand, the following result which has been proved in [5] guarantees that any function $v_{h} \in H_{h}^{K}$ is uniquely determined by the output values of the sets $\mathbf{D}_{\mathbf{1}}$ and $\mathbf{D}_{\mathbf{2}}$.

Lemma 3.1. The set of operators $\mathbf{D}_{\mathbf{1}}$ and $\mathbf{D}_{\mathbf{2}}$ constitutes a set of degrees of freedom for the space $H_{h}^{K}$.

Now, we are in a position to introduce our global virtual space for the transverse displacement and the Airy stress function:

$$
H_{h}:=\left\{v_{h} \in H_{0}^{2}(\Omega):\left.v_{h}\right|_{K} \in H_{h}^{K}\right\} .
$$

\subsection{Construction of bilinear and trilinear forms and the loading term.}

In this subsection we will propose discrete versions of the local forms to construct the discrete virtual scheme. We begin by introducing new projectors: For $\ell=0,1,2$, we consider $\Pi_{K}^{\ell}: L^{2}(K) \rightarrow \mathbb{P}_{\ell}(K)$ the standard $L^{2}$-orthogonal projector defined as follows:

$$
\int_{K} \Pi_{K}^{\ell} v q=\int_{K} v q \quad \forall q \in \mathbb{P}_{\ell}(K) \quad \forall v \in L^{2}(K) .
$$


Now, due to the particular property appearing in definition of the space $H_{h}^{K}$, it can be seen that the right hand side in (3.3) is computable using $\Pi_{K}^{2, D} v$. Thus, $\Pi_{K}^{\ell} v, \ell=0,1,2$ depends only on the values of the degrees of freedom given by the sets $\mathbf{D}_{\mathbf{1}}$ and $\mathbf{D}_{\mathbf{2}}$. Furthermore, it is easy to check that on the space $H_{h}^{K}$ the projectors $\Pi_{K}^{2}$ and $\Pi_{K}^{2, D}$ are the same operator. In fact:

$$
\int_{K}\left(\Pi_{K}^{2} v_{h}\right) q=\int_{K} v_{h} q=\int_{K}\left(\Pi_{K}^{2, D} v_{h}\right) q \quad \forall q \in \mathbb{P}_{2}(K), \quad \forall v_{h} \in H_{h}^{K} .
$$

We introduce the projector $\Pi_{K}^{2, \nabla}: H^{1}(K) \longrightarrow \mathbb{P}_{2}(K)$, for each $v \in H^{1}(K)$ as the solution of

$$
\begin{aligned}
a_{K}^{\nabla}\left(\Pi_{K}^{2, \nabla} v, q\right) v & =a_{K}^{\nabla}(v, q) \quad \forall q \in \mathbb{P}_{2}(K), \\
\int_{K} \Pi_{K}^{2, \nabla} v & =\int_{K} v .
\end{aligned}
$$

The following lemma proved in [5] establishes that operator $\Pi_{K}^{2, \nabla}$ is fully computable on the local virtual space $H_{h}^{K}$.

Lemma 3.2. The operator $\Pi_{K}^{2, \nabla}: H_{h}^{K} \rightarrow \mathbb{P}_{2}(K) \subseteq H_{h}^{K}$ is well defined and depends only on the values of the degrees of freedom given by the sets $\mathbf{D}_{\mathbf{1}}$ and $\mathbf{D}_{\mathbf{2}}$.

Following the standard procedure in VEM literature (see for instance $[2,8,24,30]$ ), we propose the following (computable) discrete local bilinear forms:

$$
a_{h, K}^{\Delta}: H_{h}^{K} \times H_{h}^{K} \rightarrow \mathbb{R} ; \quad \text { and } \quad a_{h, K}^{\nabla}: H_{h}^{K} \times H_{h}^{K} \rightarrow \mathbb{R}
$$

defined by

$$
\begin{aligned}
a_{h, K}^{\Delta}\left(u_{h}, v_{h}\right):= & \left\{\begin{array}{l}
\int_{K} \Delta \Pi_{K}^{2, D} u_{h} \Delta \Pi_{K}^{2, D} v_{h}+\bar{\alpha} s_{K}^{D}\left(u_{h}-\Pi_{K}^{2, D} u_{h}, v_{h}-\Pi_{K}^{2, D} v_{h}\right), \\
\int_{K} D^{2} \Pi_{K}^{2, D} u_{h}: D^{2} \Pi_{K}^{2, D} v_{h}+\bar{\alpha} s_{K}^{D}\left(u_{h}-\Pi_{K}^{2, D} u_{h}, v_{h}-\Pi_{K}^{2, D} v_{h}\right),
\end{array}\right. \\
a_{h, K}^{\nabla}\left(u_{h}, v_{h}\right):= & -\left\{\int_{K} \Pi_{K}^{1} \nabla u_{h} \cdot \Pi_{K}^{1} \nabla v_{h}+\widehat{\alpha} s_{K}^{\nabla}\left(u_{h}-\Pi_{K}^{2, \nabla} u_{h}, v_{h}-\Pi_{K}^{2, \nabla} v_{h}\right)\right\},
\end{aligned}
$$

respectively, where $\Pi_{K}^{1}: L^{2}(K)^{2} \rightarrow \mathbb{P}_{1}(K)^{2}$ is the standard $L^{2}$-orthogonal projector and $s_{K}^{D}(\cdot, \cdot)$ and $s_{K}^{\nabla}(\cdot, \cdot)$ are two symmetric positive definite bilinear forms satisfying the following conditions:

$$
\begin{array}{llll}
c_{0} a_{K}^{D}\left(v_{h}, v_{h}\right) \leq s_{K}^{D}\left(v_{h}, v_{h}\right) \leq c_{1} a_{K}^{D}\left(v_{h}, v_{h}\right) & \forall v_{h} \in H_{h}^{K} & \text { with } & \Pi_{K}^{2, D} v_{h}=0, \\
c_{2} a_{K}^{\nabla}\left(v_{h}, v_{h}\right) \leq s_{K}^{\nabla}\left(v_{h}, v_{h}\right) \leq c_{3} a_{K}^{\nabla}\left(v_{h}, v_{h}\right) & \forall v_{h} \in H_{h}^{K} & \text { with } & \Pi_{K}^{2, \nabla} v_{h}=0,
\end{array}
$$

respectively. In addition, in (3.6) and (3.7), $\bar{\alpha}$ and $\widehat{\alpha}$ are constants which depend on the physical parameters.

Remark 3.1. In (3.7) the vector function $\boldsymbol{\Pi}_{K}^{1} \nabla v_{h}$ is fully computable from the degrees of freedom given by the sets $\mathbf{D}_{\mathbf{1}}$ and $\mathbf{D}_{\mathbf{2}}$ (see for instance [44], Sect. 3 for further details).

Now, we define the following global discrete bilinear forms on $H_{h}$.

$$
\begin{array}{ll}
a_{h}^{\Delta}\left(u_{h}, v_{h}\right):=\sum_{K \in \mathcal{T}_{h}} a_{h, K}^{\Delta}\left(u_{h}, v_{h}\right), & u_{h}, v_{h} \in H_{h}, \\
a_{h}^{\nabla}\left(u_{h}, v_{h}\right):=\sum_{K \in \mathcal{T}_{h}} a_{h, K}^{\nabla}\left(u_{h}, v_{h}\right), & u_{h}, v_{h} \in H_{h} .
\end{array}
$$

The following result establishes the usual properties of consistency and stability for the local virtual forms. 
Proposition 3.1. The local bilinear forms $a_{h, K}^{\Delta}(\cdot, \cdot)$ and $a_{h, K}^{\nabla}(\cdot, \cdot)$ on each element $K$ satisfy

- Consistency: for all $h>0$ and for all $K \in \mathcal{T}_{h}$, we have that

$$
\begin{array}{lll}
a_{h, K}^{\Delta}\left(q, v_{h}\right)=a_{K}^{\Delta}\left(q, v_{h}\right) & \forall q \in \mathbb{P}_{2}(K), & \forall v_{h} \in H_{h}^{K}, \\
a_{h, K}^{\nabla}\left(q, v_{h}\right)=a_{K}^{\nabla}\left(q, v_{h}\right) & \forall q \in \mathbb{P}_{2}(K), & \forall v_{h} \in H_{h}^{K} .
\end{array}
$$

- Stability and boundedness: There exist positive constants $\alpha_{i}, i=1, \ldots, 4$ independent of $K$, such that:

$$
\begin{array}{ll}
\alpha_{1} a_{K}^{D}\left(v_{h}, v_{h}\right) \leq a_{h, K}^{\Delta}\left(v_{h}, v_{h}\right) \leq \alpha_{2} a_{K}^{D}\left(v_{h}, v_{h}\right) & \forall v_{h} \in H_{h}^{K}, \\
\alpha_{3} a_{K}^{\nabla}\left(v_{h}, v_{h}\right) \leq a_{h, K}^{\nabla}\left(v_{h}, v_{h}\right) \leq \alpha_{4} a_{K}^{\nabla}\left(v_{h}, v_{h}\right) & \forall v_{h} \in H_{h}^{K} .
\end{array}
$$

Proof. Since the proof can be followed from standard arguments in the Virtual Element literature (see $[2,19])$, it is omitted.

On the other hand, we will propose on each element $K$ the following local (and computable) approximation for the trilinear form $b(\cdot ; \cdot, \cdot)(c f .(2.10))$ :

$$
b_{h, K}\left(w_{h} ; u_{h}, v_{h}\right):=-\frac{1}{2} \int_{K}\left[\Pi_{K}^{2, D} w_{h}, \Pi_{K}^{2, D} u_{h}\right] \Pi_{K}^{2} v_{h} .
$$

Now, we are going to introduce the discrete version of (2.13)-(2.16). First, let us define $\mathbb{H}_{h}:=H_{h} \times H_{h}$, $\mathbb{H}_{h}^{K}:=H_{h}^{K} \times H_{h}^{K}$ and let $A_{h}^{\Delta}, A_{h}^{\nabla}, F_{h}, B_{h}$ be the discrete forms given by:

$$
\begin{array}{ll}
A_{h}^{\Delta}: \mathbb{H}_{h} \times \mathbb{H}_{h} \rightarrow \mathbb{R} ; & A_{h}^{\Delta}\left(\mathbf{u}_{h}, \mathbf{v}_{h}\right):=\sum_{K \in \mathcal{T}_{h}} A_{h, K}^{\Delta}\left(\mathbf{u}_{h}, \mathbf{v}_{h}\right) ; \\
A_{h}^{\nabla}: \mathbb{H}_{h} \times \mathbb{H}_{h} \rightarrow \mathbb{R} ; & A_{h}^{\nabla}\left(\mathbf{u}_{h}, \mathbf{v}_{h}\right):=\sum_{K \in \mathcal{T}_{h}} A_{h, K}^{\nabla}\left(\mathbf{u}_{h}, \mathbf{v}_{h}\right) ; \\
F_{h}: \mathbb{H}_{h} \rightarrow \mathbb{R} ; & F_{h}\left(\mathbf{v}_{h}\right):=\sum_{K \in \mathcal{T}_{h}} F_{h, K}\left(\mathbf{v}_{h}\right) ; \\
B_{h}: \mathbb{H}_{h} \times \mathbb{H}_{h} \times \mathbb{H}_{h} \rightarrow \mathbb{R} ; & B_{h}\left(\mathbf{w}_{h} ; \mathbf{u}_{h}, \mathbf{v}_{h}\right):=\sum_{K \in \mathcal{T}_{h}} B_{h, K}\left(\mathbf{w}_{h} ; \mathbf{u}_{h}, \mathbf{v}_{h}\right) ;
\end{array}
$$

where

$$
\begin{aligned}
A_{h, K}^{\Delta}\left(\mathbf{u}_{h}, \mathbf{v}_{h}\right) & :=a_{h, K}^{\Delta}\left(u_{h}, v_{h}\right)+a_{h, K}^{\Delta}\left(\psi_{h}, \varphi_{h}\right) \\
A_{h, K}^{\nabla}\left(\mathbf{u}_{h}, \mathbf{v}_{h}\right) & :=a_{h, K}^{\nabla}\left(u_{h}, v_{h}\right) ; \\
F_{h, K}\left(\mathbf{v}_{h}\right) & :=\int_{K} \Pi_{K}^{2} f v_{h} \equiv \int_{K} \Pi_{K}^{2} f \Pi_{K}^{2, D} v_{h} \equiv \int_{K} f \Pi_{K}^{2, D} v_{h} ; \\
B_{h, K}\left(\mathbf{w}_{h} ; \mathbf{u}_{h}, \mathbf{v}_{h}\right) & :=b_{h, K}\left(w_{h} ; \psi_{h}, v_{h}\right)+b_{h, K}\left(\xi_{h} ; u_{h}, v_{h}\right)-b_{h, K}\left(w_{h} ; u_{h}, \varphi_{h}\right) \\
& =-\frac{1}{2} \int_{K}\left\{\left[\Pi_{K}^{2, D} w_{h}, \Pi_{K}^{2, D} \psi_{h}\right] \Pi_{K}^{2} v_{h}+\left[\Pi_{K}^{2, D} \xi_{h}, \Pi_{K}^{2, D} u_{h}\right] \Pi_{K}^{2} v_{h}-\left[\Pi_{K}^{2, D} w_{h}, \Pi_{K}^{2, D} u_{h}\right] \Pi_{K}^{2} \varphi_{h}\right\},
\end{aligned}
$$

with $\mathbf{u}_{h}:=\left(u_{h}, \psi_{h}\right), \mathbf{v}_{h}:=\left(v_{h}, \varphi_{h}\right), \mathbf{w}_{h}:=\left(w_{h}, \xi_{h}\right) \in \mathbb{H}_{h}$.

Now, we are ready to propose the virtual element scheme to approximate the isolated solutions of the von Kármán problem: given $(f, \lambda) \in L^{2}(\Omega) \times \mathbb{R}$, find $\mathbf{u}_{h}:=\left(u_{h}, \psi_{h}\right) \in \mathbb{H}_{h}$ such that

$$
A_{h}^{\Delta}\left(\mathbf{u}_{h}, \mathbf{v}_{h}\right)+\lambda A_{h}^{\nabla}\left(\mathbf{u}_{h}, \mathbf{v}_{h}\right)+B_{h}\left(\mathbf{u}_{h} ; \mathbf{u}_{h}, \mathbf{v}_{h}\right)=F_{h}\left(\mathbf{v}_{h}\right) \quad \forall \mathbf{v}_{h}:=\left(v_{h}, \varphi_{h}\right) \in \mathbb{H}_{h} .
$$


In the next section we are going to prove the well posedness of the discrete problem (3.15), provided that $(f, \lambda)$ is small enough. To do that we will use a fix point argument. With this aim, we present that following discrete version of Lemma 2.1 .

Lemma 3.3. There exist positive constants $\alpha, C$ and $C_{B}$ independent of $h$ such that

$$
\begin{aligned}
\left|A_{h}^{\Delta}\left(\mathbf{u}_{h}, \mathbf{v}_{h}\right)\right| & \leq\left\|\mathbf{u}_{h}\right\|_{2, \Omega}\left\|\mathbf{v}_{h}\right\|_{2, \Omega} & & \forall \mathbf{u}_{h}, \mathbf{v}_{h} \in \mathbb{H}_{h} ; \\
\left|A_{h}^{\nabla}\left(\mathbf{u}_{h}, \mathbf{v}_{h}\right)\right| & \leq|\lambda|\left\|\mathbf{u}_{h}\right\|_{2, \Omega}\left\|\mathbf{v}_{h}\right\|_{2, \Omega} & & \forall \mathbf{u}_{h}, \mathbf{v}_{h} \in \mathbb{H}_{h} ; \\
A_{h}^{\Delta}\left(\mathbf{v}_{h}, \mathbf{v}_{h}\right) & \geq \alpha\left\|\mathbf{v}_{h}\right\|_{2, \Omega}^{2} & & \forall \mathbf{v}_{h} \in \mathbb{H}_{h} ; \\
\left|F_{h}\left(\mathbf{v}_{h}\right)\right| & \leq\|f\|_{0, \Omega}\left\|\mathbf{v}_{h}\right\|_{2, \Omega} & & \forall \mathbf{u}_{h}, \mathbf{v}_{h} \in \mathbb{H}_{h} ; \\
\left|B_{h}\left(\mathbf{w}_{h} ; \mathbf{u}_{h}, \mathbf{v}_{h}\right)\right| & \leq C\left\|\mathbf{w}_{h}\right\|_{2, \Omega}\left\|\mathbf{u}_{h}\right\|_{2, \Omega}\left\|\mathbf{v}_{h}\right\|_{2, \Omega} & & \forall \mathbf{w}_{h}, \mathbf{u}_{h}, \mathbf{v}_{h} \in \\
B_{h}\left(\mathbf{w}_{h} ; \mathbf{u}_{h}, \mathbf{v}_{h}\right) & =B_{h}\left(\mathbf{u}_{h} ; \mathbf{w}_{h}, \mathbf{v}_{h}\right) & & \forall \mathbf{w}_{h}, \mathbf{u}_{h}, \mathbf{v}_{h} \in
\end{aligned}
$$

Proof. The proof follows from (3.8) and (3.9) and Proposition 3.1.

We end this section with some definitions and results which will be used in Section 4 to prove the solvability of the discrete problem (3.15).

First, we introduce the following broken seminorm and projectors:

$$
|v|_{\ell, h}^{2}:=\sum_{K \in \mathcal{T}_{h}}|v|_{\ell, K}^{2} \quad \forall v \in L^{2}(\Omega) \text { such that }\left.v\right|_{K} \in H^{\ell}(K) \quad \ell=1,2 .
$$

Now, for all $v \in L^{2}(\Omega)$ such that $\left.v\right|_{K} \in H^{2}(K)$ for all $K \in \mathcal{T}_{h}$, we define $\Pi_{h}^{2, D}$ in $L^{2}(\Omega)$ as follows

$$
\left.\left(\Pi_{h}^{2, D} v\right)\right|_{K}:=\Pi_{K}^{2, D}\left(\left.v\right|_{K}\right) \quad \forall K \in \mathcal{T}_{h} .
$$

Next, we present the following standard approximation results. Proposition 3.2 is derived by interpolation between Sobolev spaces from the analogous result for integer values of $s$. In fact, this result for integer values is stated in Proposition 4.2 of [8] and follows from the classical Scott-Dupont theory (see [21] and [5], Prop. 3.1). Proposition 3.3 has been proved in Proposition 4.2 of [16]. Proposition 3.4 and Lemma 3.4 can be seen for instance in Section 4 of [44] and Lemma 3.5 of [45], respectively.

Proposition 3.2. If the assumption $\mathbf{A} 1$ is satisfied, then there exists a constant $C>0$, depending on the constant in assumption $\mathbf{A} 1$, such that for every $v \in H^{\delta}(K)$ there exists $v_{\pi} \in \mathbb{P}_{k}(K), k \geq 0$ such that

$$
\left\|v-v_{\pi}\right\|_{\ell, K} \leq C h_{K}^{\delta-\ell}\|v\|_{\delta, K} \quad 0 \leq \delta \leq k+1, \ell=0, \ldots,[\delta],
$$

with $[\delta]$ denoting the largest integer equal or smaller than $\delta \in \mathbb{R}$.

Proposition 3.3. Assume $\boldsymbol{A} 1$ and $\boldsymbol{A} 2$ are satisfied, let $v \in H^{2+s}(\Omega)$ with $s \in(1 / 2,1]$. Then, there exist $v_{I} \in H_{h}$ and $\widetilde{C}>0$ independent of $h$ such that

$$
\left\|v-v_{I}\right\|_{2, \Omega} \leq \widetilde{C} h^{s}\|v\|_{2+s, \Omega} .
$$

Proposition 3.4. There exists $\bar{C}>0$, independent of $h$, such that for all $\mathbf{v} \in H^{\delta}(K)^{2}$

$$
\left\|\mathbf{v}-\Pi_{K}^{1} \mathbf{v}\right\|_{0, K} \leq \bar{C} h_{K}^{\delta}\|\mathbf{v}\|_{\delta, K} \quad 0 \leq \delta \leq 2,
$$

where $\boldsymbol{\Pi}_{K}^{1}: L^{2}(K)^{2} \rightarrow \mathbb{P}_{1}(K)^{2}$ is the standard $L^{2}$-orthogonal projector (cf. Rem. 3.1).

Lemma 3.4. There exists $\widehat{C}>0$, independent of $h$, such that for every $v_{h} \in H_{h}$

$$
\left\|v_{h}-\Pi_{h}^{2, D} v_{h}\right\|_{0, \Omega} \leq \widehat{C} h^{2}\left\|v_{h}\right\|_{2, \Omega} .
$$




\section{Analysis of the Discrete PROBlem}

The purpose of this section is to prove that problem (3.15) admits a unique solution. With this end, from now on, we assume that $\mathbf{u}$ is an isolated solution of the von Kármán system (2.12).

Now, from Theorem 2.1, we know that $\mathbf{u}=(u, \psi) \in\left[H^{2+s}(\Omega)\right]^{2}$ with $s \in(1 / 2,1]$. Then, from Proposition 3.3 we have that there exists $\mathbf{u}_{I}:=\left(u_{I}, \psi_{I}\right) \in \mathbb{H}_{h}$ (from now on $\mathbf{u}_{I}$ denotes the interpolation of $\mathbf{u}$ ) such that

$$
\left\|\mathbf{u}-\mathbf{u}_{I}\right\|_{2, \Omega} \leq C h^{s}\|\mathbf{u}\|_{2+s, \Omega},
$$

where, $u_{I}$ and $\psi_{I}$ are the interpolants of $u$ and $\psi$, respectively.

In order to establish the well posedness of the discrete problem (3.15), we need to introduce some definitions. Let $A_{h, \mathbf{u}_{I}}: \mathbb{H}_{h} \times \mathbb{H}_{h} \rightarrow \mathbb{R}$ be the discrete form defined by

$$
A_{h, \mathbf{u}_{I}}\left(\mathbf{w}_{h}, \mathbf{v}_{h}\right):=A_{h}^{\Delta}\left(\mathbf{w}_{h}, \mathbf{v}_{h}\right)+\lambda A_{h}^{\nabla}\left(\mathbf{w}_{h}, \mathbf{v}_{h}\right)+2 B_{h}\left(\mathbf{u}_{I} ; \mathbf{w}_{h}, \mathbf{v}_{h}\right) \quad \forall \mathbf{w}_{h}, \mathbf{v}_{h} \in \mathbb{H}_{h} .
$$

We also define the operator

$$
\begin{aligned}
T_{h}: \mathbb{H}_{h} & \longrightarrow \mathbb{H}_{h} \\
\mathbf{w}_{h} & \longmapsto T_{h} \mathbf{w}_{h},
\end{aligned}
$$

where $T_{h} \mathbf{w}_{h} \in \mathbb{H}_{h}$ is the unique solution (to be proved below) of the following problem. Find $T_{h} \mathbf{w}_{h} \in \mathbb{H}_{h}$ such that

$$
A_{h, \mathbf{u}_{I}}\left(T_{h} \mathbf{w}_{h}, \mathbf{v}_{h}\right)=2 B_{h}\left(\mathbf{u}_{I} ; \mathbf{w}_{h}, \mathbf{v}_{h}\right)-B_{h}\left(\mathbf{w}_{h} ; \mathbf{w}_{h}, \mathbf{v}_{h}\right)+F_{h}\left(\mathbf{v}_{h}\right) \quad \forall \mathbf{v}_{h} \in \mathbb{H}_{h} .
$$

It is easy to check that any solution $\mathbf{u}_{h}$ of the discrete problem (3.15) is a fixed point of $T_{h}$ and reciprocally.

Now, we focus on proving that $T_{h}$ is well defined and then we will use contraction and fixed point arguments to establish that $T_{h}$ has a unique fixed point. To do that, we first need to prove an auxiliary lemma, which follows the argument presented in the proof of Lemma 2 from [23].

Lemma 4.1. Let $\widetilde{\mathbf{v}} \in \mathbb{H}$ such that $\|\widetilde{\mathbf{v}}\|_{2, \Omega}=1$. Then, the following problem: find $\widetilde{\mathbf{v}}_{h} \in \mathbb{H}_{h}$ such that

$$
A_{h}^{\Delta}\left(\widetilde{\mathbf{v}}_{h}, \mathbf{z}_{h}\right)=A^{\Delta}\left(\widetilde{\mathbf{v}}, \mathbf{z}_{h}\right) \quad \forall \mathbf{z}_{h} \in \mathbb{H}_{h},
$$

has a unique solution and satisfies the following a priori estimates

$$
\left\|\widetilde{\mathbf{v}}-\widetilde{\mathbf{v}}_{h}\right\|_{0, \Omega} \leq C h^{t}\|\widetilde{\mathbf{v}}\|_{2, \Omega} \quad \text { and } \quad\left\|\widetilde{\mathbf{v}}-\widetilde{\mathbf{v}}_{h}\right\|_{\infty, \Omega} \leq C h^{t / 4}\|\widetilde{\mathbf{v}}\|_{2, \Omega},
$$

where the constant $t \in(1 / 2,1]$ is the Sobolev regularity for the biharmonic equation with the right hand side in $H^{-1}(\Omega)$ and homogeneous Dirichlet boundary conditions (see e.g. [18]), and C is a positive constant independent of $h$.

Proof. We know from Lemma 2.1 that $A^{\Delta}\left(\widetilde{\mathbf{v}}, \mathbf{z}_{h}\right) \leq C\left\|\mathbf{z}_{h}\right\|_{2, \Omega}$ for all $\mathbf{z}_{h} \in \mathbb{H}_{h}$. Then, from (3.16a), (3.16c), and Lax-Milgram Lemma, we obtain that the problem (4.4) has a unique solution and satisfies the following estimate (see [21])

$$
\left\|\widetilde{\mathbf{v}}-\widetilde{\mathbf{v}}_{h}\right\|_{2, \Omega} \leq C\|\widetilde{\mathbf{v}}\|_{2, \Omega},
$$

for some positive constant $C$ independent of $h$.

On the other hand, we will use duality arguments to obtain an error bound for $\left\|\widetilde{\mathbf{v}}_{-} \widetilde{\mathbf{v}}_{h}\right\|_{0, \Omega}$. In fact, we consider the following problem: find $\mathbf{r} \in \mathbb{H}$ such that

$$
\begin{aligned}
\Delta^{2} \mathbf{r}=\widetilde{\mathbf{v}}-\widetilde{\mathbf{v}}_{h} & \text { in } \Omega, \\
\mathbf{r}=\partial_{\nu} \mathbf{r}=0 & \text { on } \partial \Omega .
\end{aligned}
$$


It is well known that problem (4.7) has a unique solution (see for instance $[18,20]$ ) and that there exists a positive constant $t \in(1 / 2,1]$ such that

$$
\|\mathbf{r}\|_{2+t, \Omega} \leq C\left\|\widetilde{\mathbf{v}}-\widetilde{\mathbf{v}}_{h}\right\|_{0, \Omega}
$$

Now, from (4.8) and Proposition 3.3 we have that there exist $\mathbf{r}_{I} \in \mathbb{H}_{h}$ and $C>0$, independent of $h$, such that

$$
\left\|\mathbf{r}-\mathbf{r}_{I}\right\|_{2, \Omega} \leq C h^{t}\|\mathbf{r}\|_{2+t, \Omega}
$$

Next, by multiplying the system (4.7) by $\widetilde{\mathbf{v}}-\widetilde{\mathbf{v}}_{h} \in \mathbb{H}$, integrating by parts twice, adding and subtracting $\mathbf{r}_{I}$, using the symmetry of $A_{h}^{\Delta}(\cdot, \cdot)$ and (4.4), we obtain

$$
\begin{aligned}
\left\|\widetilde{\mathbf{v}}-\widetilde{\mathbf{v}}_{h}\right\|_{0, \Omega}^{2} & =A^{\Delta}\left(\mathbf{r}, \widetilde{\mathbf{v}}-\widetilde{\mathbf{v}}_{h}\right)=A^{\Delta}\left(\mathbf{r}-\mathbf{r}_{I}, \widetilde{\mathbf{v}}-\widetilde{\mathbf{v}}_{h}\right)+A^{\Delta}\left(\mathbf{r}_{I}, \widetilde{\mathbf{v}}_{-} \widetilde{\mathbf{v}}_{h}\right) \\
& =A^{\Delta}\left(\mathbf{r}-\mathbf{r}_{I}, \widetilde{\mathbf{v}}-\widetilde{\mathbf{v}}_{h}\right)+A^{\Delta}\left(\widetilde{\mathbf{v}}, \mathbf{r}_{I}\right)-A^{\Delta}\left(\widetilde{\mathbf{v}}_{h}, \mathbf{r}_{I}\right) \\
& =A^{\Delta}\left(\mathbf{r}-\mathbf{r}_{I}, \widetilde{\mathbf{v}}-\widetilde{\mathbf{v}}_{h}\right)+A_{h}^{\Delta}\left(\widetilde{\mathbf{v}}_{h}, \mathbf{r}_{I}\right)-A^{\Delta}\left(\widetilde{\mathbf{v}}_{h}, \mathbf{r}_{I}\right) .
\end{aligned}
$$

Now, from (4.8) and Proposition 3.2 there exists $\mathbf{r}_{\pi} \in\left[\mathbb{P}_{2}(K)\right]^{2}$ such that

$$
\left|\mathbf{r}-\mathbf{r}_{\pi}\right|_{2, K} \leq C h^{t}|\mathbf{r}|_{2+t, K} \quad \forall K \in \mathcal{T}_{h} .
$$

Thus, adding and subtracting $\mathbf{r}_{\pi}$, and using the consistency property (3.10) on the right hand side of (4.10), we obtain that

$$
\left\|\widetilde{\mathbf{v}}-\widetilde{\mathbf{v}}_{h}\right\|_{0, \Omega}^{2}=A^{\Delta}\left(\mathbf{r}-\mathbf{r}_{I}, \widetilde{\mathbf{v}}-\widetilde{\mathbf{v}}_{h}\right)+\sum_{K \in \mathcal{T}_{h}}\left\{A_{h, K}^{\Delta}\left(\widetilde{\mathbf{v}}_{h}, \mathbf{r}_{I}-\mathbf{r}_{\pi}\right)+A_{K}^{\Delta}\left(\widetilde{\mathbf{v}}_{h}, \mathbf{r}_{\pi}-\mathbf{r}_{I}\right)\right\} .
$$

Next, using Lemma 2.1, Cauchy-Schwarz inequality, Poincaré inequality, (3.12) and finally adding and subtracting $\mathbf{r}$ on the right hand side of the above term, we obtain

$$
\begin{aligned}
\left\|\widetilde{\mathbf{v}}-\widetilde{\mathbf{v}}_{h}\right\|_{0, \Omega}^{2} \leq & C\left\|\mathbf{r}-\mathbf{r}_{I}\right\|_{2, \Omega}\left\|\widetilde{\mathbf{v}}-\widetilde{\mathbf{v}}_{h}\right\|_{2, \Omega}+\sum_{K \in \mathcal{T}_{h}}\left\{A_{h, K}^{\Delta}\left(\widetilde{\mathbf{v}}_{h}, \widetilde{\mathbf{v}}_{h}\right)^{1 / 2} A_{h, K}^{\Delta}\left(\mathbf{r}_{I}-\mathbf{r}_{\pi}, \mathbf{r}_{I}-\mathbf{r}_{\pi}\right)^{1 / 2}\right. \\
& \left.+A_{K}^{\Delta}\left(\widetilde{\mathbf{v}}_{h}, \widetilde{\mathbf{v}}_{h}\right)^{1 / 2} A_{K}^{\Delta}\left(\mathbf{r}_{I}-\mathbf{r}_{\pi}, \mathbf{r}_{I}-\mathbf{r}_{\pi}\right)^{1 / 2}\right\} \\
\leq & C\left\|\mathbf{r}-\mathbf{r}_{I}\right\|_{2, \Omega}\left\|\widetilde{\mathbf{v}}-\widetilde{\mathbf{v}}_{h}\right\|_{2, \Omega}+\left(\alpha_{2}+1\right) \sum_{K \in \mathcal{T}_{h}}\left|\widetilde{\mathbf{v}}_{h}\right|_{2, K}\left|\mathbf{r}_{I}-\mathbf{r}_{\pi}\right|_{2, K} \\
\leq & C\left\|\mathbf{r}-\mathbf{r}_{I}\right\|_{2, \Omega}\left\|\widetilde{\mathbf{v}}-\widetilde{\mathbf{v}}_{h}\right\|_{2, \Omega}+\left(\alpha_{2}+1\right) \sum_{K \in \mathcal{T}_{h}}\left|\widetilde{\mathbf{v}}_{h}\right|_{2, K}\left(\left|\mathbf{r}-\mathbf{r}_{\pi}\right|_{2, K}+\left|\mathbf{r}-\mathbf{r}_{I}\right|_{2, K}\right) .
\end{aligned}
$$

Then, using (4.9), (4.6), (4.11), and (4.8) in the above term, we infer

$$
\left\|\widetilde{\mathbf{v}}-\widetilde{\mathbf{v}}_{h}\right\|_{0, \Omega}^{2} \leq C h^{t}\|\mathbf{r}\|_{2+t, \Omega}\|\widetilde{\mathbf{v}}\|_{2, \Omega} \leq C h^{t}\left\|\widetilde{\mathbf{v}}-\widetilde{\mathbf{v}}_{h}\right\|_{0, \Omega}\|\widetilde{\mathbf{v}}\|_{2, \Omega},
$$

and therefore, we conclude that

$$
\left\|\widetilde{\mathbf{v}}-\widetilde{\mathbf{v}}_{h}\right\|_{0, \Omega} \leq C h^{t}\|\widetilde{\mathbf{v}}\|_{2, \Omega}
$$

Now, using interpolation theory ([21], Chap. 14) on the estimates (4.6) and (4.12), we obtain that for all $\delta \in[0,2]$ the following estimate holds true

$$
\left\|\widetilde{\mathbf{v}}-\widetilde{\mathbf{v}}_{h}\right\|_{\delta, \Omega} \leq C h^{t(2-\delta) / 2}\|\widetilde{\mathbf{v}}\|_{2, \Omega} .
$$

Then, using the Sobolev injection of $H^{1+\sigma}(\Omega)$ (for all $\sigma>0$ ) in $L^{\infty}(\Omega)$ (see e.g. [1], Thm. 4.12) we have in particular that

$$
\left\|\widetilde{\mathbf{v}}-\widetilde{\mathbf{v}}_{h}\right\|_{\infty, \Omega} \leq C\left\|\widetilde{\mathbf{v}}-\widetilde{\mathbf{v}}_{h}\right\|_{\tilde{\delta}, \Omega} \leq C h^{t(2-\tilde{\delta}) / 2}\|\widetilde{\mathbf{v}}\|_{2, \Omega}
$$

for all $\tilde{\delta} \in(1,2]$. In particular, taking $\tilde{\delta}=3 / 2$, we conclude the proof. 
The next step is to establish a technical result for the trilinear forms $B_{h}(\cdot ; \cdot, \cdot)$ and $B(\cdot ; \cdot, \cdot)$.

Lemma 4.2. Let $\mathbf{u}=(u, \psi) \in \mathbb{H}$ be an isolated solution of problem (2.12), and let $\widetilde{\mathbf{v}} \in \mathbb{H}$ be such that $\|\widetilde{\mathbf{v}}\|_{2, \Omega}=1$. Then, there exists a positive constant $C$, independent of $h$, such that

$$
B_{h}\left(\mathbf{u}_{I} ; \mathbf{w}_{h}, \widetilde{\mathbf{v}}_{h}\right)-B\left(\mathbf{u}_{I} ; \mathbf{w}_{h}, \widetilde{\mathbf{v}}\right) \leq C h^{\tilde{s}}\|\mathbf{u}\|_{2+s, \Omega}\left\|\mathbf{w}_{h}\right\|_{2, \Omega} \quad \forall \mathbf{w}_{h} \in \mathbb{H}_{h},
$$

with $\tilde{s}:=\min \{s, t / 4\}$ for $s, t \in(1 / 2,1]$ and where $\widetilde{\mathbf{v}}_{h} \in \mathbb{H}_{h}$ and $t$ are such that Lemma 4.1 holds true.

Proof. We start by adding and subtracting the term $B\left(\mathbf{u}_{I} ; \mathbf{w}_{h}, \widetilde{\mathbf{v}}_{h}\right)$ to obtain

$$
\begin{aligned}
B_{h}\left(\mathbf{u}_{I} ; \mathbf{w}_{h}, \widetilde{\mathbf{v}}_{h}\right)-B\left(\mathbf{u}_{I} ; \mathbf{w}_{h}, \widetilde{\mathbf{v}}\right) & =\left\{B_{h}\left(\mathbf{u}_{I} ; \mathbf{w}_{h}, \widetilde{\mathbf{v}}_{h}\right)-B\left(\mathbf{u}_{I} ; \mathbf{w}_{h}, \widetilde{\mathbf{v}}_{h}\right)\right\}+B\left(\mathbf{u}_{I} ; \mathbf{w}_{h}, \widetilde{\mathbf{v}}_{h}-\widetilde{\mathbf{v}}\right) \\
& =B\left(\mathbf{u}_{I} ; \mathbf{w}_{h}, \widetilde{\mathbf{v}}_{h}-\widetilde{\mathbf{v}}\right)+\left\{B_{h}\left(\mathbf{u}_{I} ; \mathbf{w}_{h}, \widetilde{\mathbf{v}}_{h}\right)-B\left(\mathbf{u}_{I} ; \mathbf{w}_{h}, \widetilde{\mathbf{v}}_{h}\right)\right\} .
\end{aligned}
$$

Now, for first term on the right hand side above we have the following estimate,

$$
B\left(\mathbf{u}_{I} ; \mathbf{w}_{h}, \widetilde{\mathbf{v}}_{h}-\widetilde{\mathbf{v}}\right)=\int_{\Omega}\left[\mathbf{u}_{I}, \mathbf{w}_{h}\right]\left(\widetilde{\mathbf{v}}_{h}-\widetilde{\mathbf{v}}\right) \leq C\left\|\mathbf{u}_{I}\right\|_{2, \Omega}\left\|\mathbf{w}_{h}\right\|_{2, \Omega}\left\|\widetilde{\mathbf{v}}_{h}-\widetilde{\mathbf{v}}\right\|_{\infty, \Omega} \leq C h^{t / 4}\left\|\mathbf{u}_{I}\right\|_{2, \Omega}\left\|\mathbf{w}_{h}\right\|_{2, \Omega},
$$

with, $t \in(1 / 2,1]$ and where we have used the fact that $\left(\widetilde{\mathbf{v}}-\widetilde{\mathbf{v}}_{h}\right) \in L^{\infty}(\Omega)$ and estimate (4.5).

On the other hand, for the second term on right hand side of $(4.13)$, we use the definitions of $B_{h}(\cdot ; \cdot, \cdot)$ and $B(\cdot ; \cdot, \cdot)$ to get

$$
\begin{aligned}
& B_{h}\left(\mathbf{u}_{I} ; \mathbf{w}_{h}, \widetilde{\mathbf{v}}_{h}\right)-B\left(\mathbf{u}_{I} ; \mathbf{w}_{h}, \widetilde{\mathbf{v}}_{h}\right) \\
&=-\frac{1}{2} \sum_{K \in \mathcal{T}_{h}} \int_{K}\left\{\left(\left[\Pi_{K}^{2, D} u_{I}, \Pi_{K}^{2, D} \xi_{h}\right] \Pi_{K}^{2} \widetilde{v}_{h}-\left[u_{I}, \xi_{h}\right] \widetilde{v}_{h}\right)+\left(\left[\Pi_{K}^{2, D} \psi_{I}, \Pi_{K}^{2, D} w_{h}\right] \Pi_{K}^{2} \widetilde{v}_{h}-\left[\psi_{I}, w_{h}\right] \widetilde{v}_{h}\right)\right. \\
&\left.-\left(\left[\Pi_{K}^{2, D} u_{I}, \Pi_{K}^{2, D} w_{h}\right] \Pi_{K}^{2} \widetilde{\varphi}_{h}-\left[u_{I}, w_{h}\right] \widetilde{\varphi}_{h}\right)\right\}=:-\frac{1}{2} \sum_{K \in \mathcal{T}_{h}} \int_{K}\left\{\mathbf{B}^{1, K}+\mathbf{B}^{2, K}-\mathbf{B}^{3, K}\right\} .
\end{aligned}
$$

Now, we will prove that the terms $\sum_{K \in \mathcal{T}_{h}} \int_{K} \mathbf{B}^{1, K}, \sum_{K \in \mathcal{T}_{h}} \int_{K} \mathbf{B}^{2, K}$ and $\sum_{K \in \mathcal{T}_{h}} \int_{K}\left(-\mathbf{B}^{3, K}\right)$ are bounded. Indeed, for $\sum_{K \in \mathcal{T}_{h}} \int_{K} \mathbf{B}^{1, K}$, we use the definitions of $\Pi_{K}^{2}$ and $[\cdot, \cdot]$ (cf. (3.3) and (2.2), respectively) to obtain

$$
\begin{aligned}
\sum_{K \in \mathcal{T}_{h}} \int_{K} \mathbf{B}^{1, K}= & \sum_{K \in \mathcal{T}_{h}} \int_{K}\left(\left[\Pi_{K}^{2, D} u_{I}, \Pi_{K}^{2, D} \xi_{h}\right]-\left[u_{I}, \xi_{h}\right]\right) \widetilde{v}_{h} \\
= & \sum_{K \in \mathcal{T}_{h}} \int_{K}\left\{\left\{\left(\partial_{x x} \Pi_{K}^{2, D} u_{I}\right)\left(\partial_{y y} \Pi_{K}^{2, D} \xi_{h}\right) \widetilde{v}_{h}-\left(\partial_{x x} u_{I}\right)\left(\partial_{y y} \xi_{h}\right) \widetilde{v}_{h}\right\}\right. \\
& +\left\{\left(\partial_{y y} \Pi_{K}^{2, D} u_{I}\right)\left(\partial_{x x} \Pi_{K}^{2, D} \xi_{h}\right) \widetilde{v}_{h}-\left(\partial_{y y} u_{I}\right)\left(\partial_{x x} \xi_{h}\right) \widetilde{v}_{h}\right\} \\
& \left.-2\left\{\left(\partial_{x y} \Pi_{K}^{2, D} u_{I}\right)\left(\partial_{x y} \Pi_{K}^{2, D} \xi_{h}\right) \widetilde{v}_{h}-\left(\partial_{x y} u_{I}\right)\left(\partial_{x y} \xi_{h}\right) \widetilde{v}_{h}\right\}\right\}=: \sum_{K \in \mathcal{T}_{h}} \int_{K}\{\boldsymbol{\alpha}+\boldsymbol{\beta}-2 \boldsymbol{\gamma}\}
\end{aligned}
$$

Next, we will estimate the term $\sum_{K \in \mathcal{T}_{h}} \int_{K} \boldsymbol{\alpha}$. The terms $\sum_{K \in \mathcal{T}_{h}} \int_{K} \boldsymbol{\beta}$ and $\sum_{K \in \mathcal{T}_{h}} \int_{K}(-2) \boldsymbol{\gamma}$ can be treated with the same arguments. Thus, applying the identity $\partial_{i j} \Pi_{K}^{2, D} v_{h}=\Pi_{K}^{0}\left(\partial_{i j} v_{h}\right)$ for all $v_{h} \in H_{h}^{K}$ and for all $i, j=x, y$, (which is a consequence of the definitions of the projectors $\Pi_{K}^{2, D}$ and $\Pi_{K}^{0}(c f$. (3.1a) and (3.3), respectively)) in the expression $\sum_{K \in \mathcal{T}_{h}} \int_{K} \boldsymbol{\alpha}$, we obtain

$$
\sum_{K \in \mathcal{T}_{h}} \int_{K} \boldsymbol{\alpha}=\sum_{K \in \mathcal{T}_{h}} \int_{K}\left\{\Pi_{K}^{0}\left(\partial_{x x} u_{I}\right) \Pi_{K}^{0}\left(\partial_{y y} \xi_{h}\right) \widetilde{v}_{h}-\left(\partial_{x x} u_{I}\right)\left(\partial_{y y} \xi_{h}\right) \widetilde{v}_{h}\right\}
$$


Now, adding and subtracting the terms $\Pi_{K}^{0}\left(\partial_{x x} u\right) \Pi_{K}^{0}\left(\partial_{y y} \xi_{h}\right) \widetilde{v}_{h}, \Pi_{K}^{0}\left(\partial_{x x} u\right)\left(\partial_{y y} \xi_{h}\right) \widetilde{v}_{h}$ and $\left(\partial_{y y} \xi_{h}\right)\left(\partial_{x x} u\right) \widetilde{v}_{h}$, and using the definitions of $\Pi_{K}^{0}$ on the right hand side of (4.17), we get

$$
\begin{aligned}
\sum_{K \in \mathcal{T}_{h}} \int_{K} \boldsymbol{\alpha}= & \sum_{K \in \mathcal{T}_{h}} \int_{K}\left\{\left\{\Pi_{K}^{0}\left(\partial_{x x}\left(u_{I}-u\right)\right)\right\} \Pi_{K}^{0}\left(\partial_{y y} \xi_{h}\right) \widetilde{v}_{h}+\Pi_{K}^{0}\left(\partial_{x x} u\right)\left\{\left(\Pi_{K}^{0}-I\right)\left(\partial_{y y} \xi_{h}\right)\right\} \widetilde{v}_{h}\right. \\
& \left.+\left(\partial_{y y} \xi_{h}\right)\left\{\left(\Pi_{K}^{0}-I\right)\left(\partial_{x x} u\right)\right\} \widetilde{v}_{h}+\left(\partial_{y y} \xi_{h}\right)\left(\partial_{x x}\left(u-u_{I}\right)\right) \widetilde{v}_{h}\right\} \\
= & \sum_{K \in \mathcal{T}_{h}} \int_{K}\left\{\left\{\Pi_{K}^{0}\left(\partial_{x x}\left(u_{I}-u\right)\right)\right\} \Pi_{K}^{0}\left(\partial_{y y} \xi_{h}\right) \widetilde{v}_{h}+\Pi_{K}^{0}\left(\partial_{x x} u\right)\left\{\left(\Pi_{K}^{0}-I\right)\left(\partial_{y y} \xi_{h}\right)\right\}\left\{\left(I-\Pi_{K}^{0}\right) \widetilde{v}_{h}\right\}\right. \\
& \left.+\left(\partial_{y y} \xi_{h}\right)\left\{\left(\Pi_{K}^{0}-I\right)\left(\partial_{x x} u\right)\right\} \widetilde{v}_{h}+\left(\partial_{y y} \xi_{h}\right)\left(\partial_{x x}\left(u-u_{I}\right)\right) \widetilde{v}_{h}\right\} .
\end{aligned}
$$

Then, applying Cauchy-Schwarz and Hölder inequalities, using the fact that $\widetilde{v}_{h} \in L^{\infty}(K)$, and $\Pi_{K}^{0}$ is bounded in the $L^{2}$-norm, on the right hand side of the last equality, we obtain

$$
\begin{aligned}
\sum_{K \in \mathcal{T}_{h}} \int_{K} \boldsymbol{\alpha} \leq & \sum_{K \in \mathcal{T}_{h}}\left\{\left\|\Pi_{K}^{0}\left(\partial_{x x}\left(u_{I}-u\right)\right)\right\|_{0, K}\left\|\Pi_{K}^{0}\left(\partial_{y y} \xi_{h}\right)\right\|_{0, K}\left\|\widetilde{v}_{h}\right\|_{\infty, K}\right. \\
& +\left.\left\|\Pi_{K}^{0}\left(\partial_{x x} u\right)\right\|\right|_{L^{4}(K)}\left\|\left(\Pi_{K}^{0}-I\right)\left(\partial_{y y} \xi_{h}\right)\right\|_{0, K}\left\|\left(I-\Pi_{K}^{0}\right) \widetilde{v}_{h}\right\|_{L^{4}(K)} \\
& \left.+\left\|\partial_{y y} \xi_{h} \mid\right\|_{0, K}\left\|\left(\Pi_{K}^{0}-I\right)\left(\partial_{x x} u\right)\right\|\left\|_{0, K}\right\| \widetilde{v}_{h}\left\|_{\infty, K}+\right\| \partial_{y y} \xi_{h}\left\|_{0, K}\right\| \partial_{x x}\left(u-u_{I}\right)\left\|_{0, K}\right\| \widetilde{v}_{h} \|_{\infty, K}\right\} \\
\leq & \sum_{K \in \mathcal{T}_{h}}\left\{C h_{K}^{s}|u|_{2+s, K}\left|\xi_{h}\right|_{2, K}\right\}+\sum_{K \in \mathcal{T}_{h}}\left\{\left\|\Pi_{K}^{0}\left(\partial_{x x} u\right)\right\|_{L^{4}(K)}\left|\xi_{h}\right|_{2, K}\left\|\left(I-\Pi_{K}^{0}\right) \widetilde{v}_{h}\right\|_{L^{4}(K)}\right\},
\end{aligned}
$$

where in the last step, we have used Theorem 2.1, Propositions 3.3 and 3.2, and the fact that $\left\|\widetilde{v}_{h}\right\|_{\infty, K} \leq$ $\left\|\widetilde{v}_{h}\right\|_{\infty, \Omega} \leq C|| \widetilde{v}_{h}\left\|_{2, \Omega} \leq C|| \widetilde{\mathbf{v}}_{h}\right\|_{2, \Omega} \leq C\|\widetilde{\mathbf{v}}\|_{2, \Omega}=C$ (which is a consequence of the fact that $H^{1+\sigma}(\Omega) \subseteq L^{\infty}(\Omega)$ for all $\sigma>0$, see e.g. [1], Thm. 4.12).

Now, to bound the second term on the right hand side of $(4.18)$, it is easy to check that $\left\|\Pi_{K}^{0}\left(\partial_{x x} u\right)\right\|_{L^{4}(K)} \leq$ $C\left\|\partial_{x x} u\right\|_{L^{4}(K)}$ (see [15], Prop. 3.3). Thus, applying Hölder inequality (for sequences), Lemma 3.7 of [35], and using the Sobolev embeddings $H^{s}(\Omega) \hookrightarrow L^{4}(\Omega)$ for $s \in(1 / 2,1]$ and $H^{2}(\Omega) \hookrightarrow W^{1,4}(\Omega)$ (see [7], Thm. 7.3.7(a-b)), we get

$$
\begin{aligned}
\sum_{K \in \mathcal{T}_{h}} \int_{K} \boldsymbol{\alpha} \leq & C h^{s}|| u \|_{2+s, \Omega}\left|\xi_{h}\right|_{2, \Omega}+\sum_{K \in \mathcal{T}_{h}}\left\{\left\|\partial_{x x} u\right\|_{L^{4}(K)}\left|\xi_{h}\right|_{2, K}\left\|\left(I-\Pi_{K}^{0}\right) \widetilde{v}_{h}\right\|_{L^{4}(K)}\right\} \\
\leq & C h^{s}\|u\|_{2+s, \Omega}\left|\xi_{h}\right|_{2, \Omega} \\
& +\left(\sum_{K \in \mathcal{T}_{h}}\left\|\partial_{x x} u\right\|_{L^{4}(K)}^{4}\right)^{1 / 4}\left(\sum_{K \in \mathcal{T}_{h}}\left\|\partial_{y y} \xi_{h}\right\|_{0, K}^{2}\right)^{1 / 2}\left(\sum_{K \in \mathcal{T}_{h}}\left\|\left(I-\Pi_{K}^{0}\right) \widetilde{v}_{h}\right\|_{L^{4}(K)}^{4}\right)^{1 / 4} \\
\leq & C h^{s}|| u\left\|_{2+s, \Omega}\left|\xi_{h}\right|_{2, \Omega}+\right\| \partial_{x x} u\left\|_{L^{4}(\Omega)}\right\| \partial_{y y} \xi_{h} \|_{0, \Omega}\left(C \sum_{K \in \mathcal{T}_{h}} h_{K}^{4}\left|\widetilde{v}_{h}\right|_{W^{1,4}(K)}^{4}\right)^{1 / 4} \\
\leq & C h^{s}|| u\left\|_{2+s, \Omega}\left|\xi_{h}\right|_{2, \Omega}+C h\right\| \partial_{x x} u\left\|_{L^{4}(\Omega)}\right\| \partial_{y y} \xi_{h} \|_{0, \Omega}\left|\widetilde{v}_{h}\right|_{W^{1,4}(\Omega)} \\
\leq & C h^{s}\|u\|_{2+s, \Omega}|| \xi_{h}\left\|_{2, \Omega}+C h\right\| u\left\|_{2+s, \Omega}\right\| \xi_{h}\left\|_{2, \Omega}\right\| \widetilde{v}_{h}\left\|_{2, \Omega} \leq C h^{s}\right\| u\left\|_{2+s, \Omega}\right\| \xi_{h} \|_{2, \Omega}
\end{aligned}
$$

where, in the two last steps we have used the definition of norm $\|\cdot\| \|_{2, \Omega}$ and the fact that $\left\|\widetilde{v}_{h}\right\|_{2, \Omega} \leq\left\|\widetilde{\mathbf{v}}_{h}\right\|_{2, \Omega} \leq$ $C\|\widetilde{\mathbf{v}}\|_{2, \Omega}=C$. Note that $C$ is independent of the mesh parameter $h$.

Moreover, repeating the same steps used in (4.17)-(4.19), we can prove the following estimates

$$
\sum_{K \in \mathcal{T}_{h}} \int_{K} \boldsymbol{\beta} \leq C h^{s}\left\|\xi_{h}\right\|_{2, \Omega}\|u\|_{2+s, \Omega} \quad \text { and } \quad \sum_{K \in \mathcal{T}_{h}} \int_{K}(-2) \boldsymbol{\gamma} \leq C h^{s}\left\|\xi_{h}\right\|_{2, \Omega}\|u\|_{2+s, \Omega}
$$


Hence, from (4.16) and (4.18), (4.20), we obtain

$$
\sum_{K \in \mathcal{T}_{h}} \int_{K} \mathbf{B}^{1, K} \leq C h^{s}\|u\|_{2+s, \Omega}\left\|\xi_{h}\right\|_{2, \Omega}
$$

Now, we observe that the terms in (4.15) can be bounded repeating the same arguments used to bound $\sum_{K \in \mathcal{T}_{h}} \mathbf{B}^{1, K}$. Thus,

$$
\sum_{K \in \mathcal{T}_{h}} \int_{K} \mathbf{B}^{2, K} \leq C h^{s}\|\psi\|_{2+s, \Omega}\left\|w_{h}\right\|_{2, \Omega} \quad \text { and } \quad \sum_{K \in \mathcal{T}_{h}} \int_{K}(-1) \mathbf{B}^{3, K} \leq C h^{s}\|u\|_{2+s, \Omega}\left\|w_{h}\right\|_{2, \Omega}
$$

As a consequence, we have that

$$
\sum_{K \in \mathcal{T}_{h}}\left\{\int_{K} \mathbf{B}^{2, K}-\int_{K} \mathbf{B}^{3, K}\right\} \leq C h^{s}\left\{\|\psi\|_{2+s, \Omega}\left\|w_{h}\right\|_{2, \Omega}+\|u\|_{2+s, \Omega}\left\|w_{h}\right\|_{2, \Omega}\right\} .
$$

Finally, the proof follows from (4.14), (4.21) and (4.22).

Now, we will use Lemmas 4.1 and 4.2 to prove that operator $T_{h}$ is well defined. More precisely, we will show in the following result that $A_{h, \mathbf{u}_{I}}(\cdot, \cdot)$ is non singular $(c f .(2.18))$ on $\mathbb{H}_{h} \times \mathbb{H}_{h}$.

Lemma 4.3. Let $\mathbf{u}=(u, \psi) \in \mathbb{H}$ be an isolated solution of problem (2.12). Then, for $h$ small enough we have that the bilinear form $A_{h, \mathbf{u}_{I}}(\cdot, \cdot)$ (cf. (4.2)) is non singular on $\mathbb{H}_{h} \times \mathbb{H}_{h}$.

Proof. Since the discrete space $\mathbb{H}_{h} \subseteq \mathbb{H}$ we will proceed as in Lemma 2 of [23]. However, we have to deal with the approximation of the bilinear and trilinear forms in our case. We recall that at the discrete level it is enough to verify one of the two inequality in (2.18) for bilinear form $A_{h, \mathbf{u}_{I}}(\cdot, \cdot)$. We will prove that there exists a constant $C>0$, independent of $h$, such that

$$
\sup _{\substack{\mathbf{v}_{h} \in \mathbb{H}_{h} \\\left\|\mathbf{v}_{h}\right\|_{2, \Omega}=1}} A_{h, \mathbf{u}_{I}}\left(\mathbf{w}_{h}, \mathbf{v}_{h}\right) \geq C\left\|\mathbf{w}_{h}\right\|_{2, \Omega} \quad \forall \mathbf{w}_{h} \in \mathbb{H}_{h}
$$

Indeed, because of $\mathbf{u}$ is isolated, we have from Definition 2.2 that $A_{\mathbf{u}}(\cdot, \cdot)(c f .(2.17))$ is non singular on $\mathbb{H} \times \mathbb{H}$. Then, the following result is a consequence of the fact that $A_{\mathbf{u}}(\cdot, \cdot)$ is non singular, $\mathbf{u}_{I}, \mathbf{w}_{h} \in \mathbb{H}_{h} \subseteq \mathbb{H},(4.1)$ and Theorem 2.4.

$$
\sup _{\substack{\mathbf{v} \in \mathbb{H} \\\|\mathbf{v}\|_{2, \Omega}=1}} A_{\mathbf{u}_{I}}\left(\mathbf{w}_{h}, \mathbf{v}\right) \geq \bar{c}_{1}\left\|\mathbf{w}_{h}\right\|_{2, \Omega} \quad \forall \mathbf{w}_{h}:=\left(w_{h}, \xi_{h}\right) \in \mathbb{H}_{h}
$$

Next, we can choose $\widetilde{\mathbf{v}}:=(\widetilde{v}, \tilde{\varphi}) \in \mathbb{H}$ with $\|\tilde{\mathbf{v}}\|_{2, \Omega}=1$ such that

$$
A_{\mathbf{u}_{I}}\left(\mathbf{w}_{h}, \tilde{\mathbf{v}}\right) \geq \bar{c}_{1}\left\|\mathbf{w}_{h}\right\|_{2, \Omega} .
$$

Moreover, from Lemma 4.1 we have that: given $\widetilde{\mathbf{v}}=(\widetilde{v}, \tilde{\varphi})$, there exists $\widetilde{\mathbf{v}}_{h}:=\left(\widetilde{v}_{h}, \widetilde{\varphi}_{h}\right)$ and $t \in(1 / 2,1]$ such that

$$
\left\|\widetilde{v}-\widetilde{v}_{h}\right\|_{0, \Omega} \leq\left\|\widetilde{\mathbf{v}}-\widetilde{\mathbf{v}}_{h}\right\|_{0, \Omega} \leq C h^{t},
$$

and

$$
\left\|\widetilde{\mathbf{v}}-\widetilde{\mathbf{v}}_{h}\right\|_{\infty, \Omega} \leq C h^{t / 4}
$$


Now, from the left hand side of (4.23), normalizing $\widetilde{\mathbf{v}}_{h}$, using the definition of $A_{h, \mathbf{u}_{I}}(\cdot, \cdot)$ in $(4.3)$ and $A_{\mathbf{u}_{I}}(\cdot, \cdot)$ in (2.17), we obtain

$$
\begin{aligned}
\sup _{\substack{\mathbf{v}_{h} \in \mathbb{H}_{h} \\
\left\|\mathbf{v}_{h}\right\|_{2, \Omega}=1}} A_{h, \mathbf{u}_{I}}\left(\mathbf{w}_{h}, \mathbf{v}_{h}\right) & \geq A_{h, \mathbf{u}_{I}}\left(\mathbf{w}_{h}, \widetilde{\mathbf{v}}_{h}\right)=A_{\mathbf{u}_{I}}\left(\mathbf{w}_{h}, \widetilde{\mathbf{v}}\right)+\left\{A_{h, \mathbf{u}_{I}}\left(\mathbf{w}_{h}, \widetilde{\mathbf{v}}_{h}\right)-A_{\mathbf{u}_{I}}\left(\mathbf{w}_{h}, \widetilde{\mathbf{v}}\right)\right\} \\
& =A_{\mathbf{u}_{I}}\left(\mathbf{w}_{h}, \widetilde{\mathbf{v}}\right)+\lambda\left\{A_{h}^{\nabla}\left(\mathbf{w}_{h}, \widetilde{\mathbf{v}}_{h}\right)-A^{\nabla}\left(\mathbf{w}_{h}, \widetilde{\mathbf{v}}\right)\right\}+2\left\{B_{h}\left(\mathbf{u}_{I} ; \mathbf{w}_{h}, \widetilde{\mathbf{v}}_{h}\right)-B\left(\mathbf{u}_{I} ; \mathbf{w}_{h}, \widetilde{\mathbf{v}}\right)\right\} \\
& \geq \bar{c}_{1}\left\|\mathbf{w}_{h}\right\|_{2, \Omega}+\lambda\{\underbrace{A_{h}^{\nabla}\left(\mathbf{w}_{h}, \widetilde{\mathbf{v}}_{h}\right)-A^{\nabla}\left(\mathbf{w}_{h}, \widetilde{\mathbf{v}}\right)}_{E_{1}}\}+2\{\underbrace{B_{h}\left(\mathbf{u}_{I} ; \mathbf{w}_{h}, \widetilde{\mathbf{v}}_{h}\right)-B\left(\mathbf{u}_{I} ; \mathbf{w}_{h}, \widetilde{\mathbf{v}}\right)}_{E_{2}}\},
\end{aligned}
$$

where, in the last step we have used (4.24). We note that the terms $E_{1}$ and $E_{2}$ have appeared because of the approximation of the bilinear and trilinear forms. Moreover, the term $E_{2}$ has been bounded in Lemma 4.2.

In what follows, we will bound the terms $E_{1}$. Indeed, we use the definition of $\boldsymbol{\Pi}_{K}^{1}$, add and subtract the term $\nabla w_{h} \cdot \nabla \widetilde{v}_{h}$ and integrate by parts to obtain

$$
\begin{aligned}
E_{1} & =\sum_{K \in \mathcal{T}_{h}}\left\{\int_{K}\left\{\nabla w_{h} \cdot \nabla \widetilde{v}-\Pi_{K}^{1} \nabla w_{h} \cdot \Pi_{K}^{1} \nabla \widetilde{v}_{h}\right\}+s_{K}^{\nabla}\left(w_{h}-\Pi_{K}^{2, \nabla} w_{h}, \widetilde{v}_{h}-\Pi_{K}^{2, \nabla} \widetilde{v}_{h}\right)\right\} \\
& =\sum_{K \in \mathcal{T}_{h}}\left\{\int_{K} \nabla w_{h} \cdot\left\{\nabla \widetilde{v}-\Pi_{K}^{1} \nabla \widetilde{v}_{h}\right\}+s_{K}^{\nabla}\left(w_{h}-\Pi_{K}^{2, \nabla} w_{h}, \widetilde{v}_{h}-\Pi_{K}^{2, \nabla} \widetilde{v}_{h}\right)\right\} \\
& =\sum_{K \in \mathcal{T}_{h}} \int_{K}\left\{\nabla w_{h} \cdot\left\{\nabla \widetilde{v}_{h}-\Pi_{K}^{1} \nabla \widetilde{v}_{h}\right\}+\nabla w_{h} \cdot \nabla\left(\widetilde{v}-\widetilde{v}_{h}\right)+s_{K}^{\nabla}\left(w_{h}-\Pi_{K}^{2, \nabla} w_{h}, \widetilde{v}_{h}-\Pi_{K}^{2, \nabla} \widetilde{v}_{h}\right)\right\} \\
& =\sum_{K \in \mathcal{T}_{h}} \int_{K}\left\{\nabla w_{h} \cdot\left\{\nabla \widetilde{v}_{h}-\Pi_{K}^{1} \nabla \widetilde{v}_{h}\right\}+s_{K}^{\nabla}\left(w_{h}-\Pi_{K}^{2, \nabla} w_{h}, \widetilde{v}_{h}-\Pi_{K}^{2, \nabla} \widetilde{v}_{h}\right)\right\}+\int_{\Omega} \Delta w_{h}\left(\widetilde{v}_{h}-\widetilde{v}\right) .
\end{aligned}
$$

Next, applying Cauchy-Schwarz inequality three times, using (3.9) and the definition of $\Pi_{K}^{2, \nabla}$ on the right hand side above, we get

$$
\begin{aligned}
E_{1} \leq & \sum_{K \in \mathcal{T}_{h}}\left\{\left|w_{h}\right|_{1, K}|| \boldsymbol{\Pi}_{K}^{1} \nabla \widetilde{v}_{h}-\nabla \widetilde{v}_{h} \|_{0, K}+s_{K}^{\nabla}\left(w_{h}-\Pi_{K}^{2, \nabla} w_{h}, w_{h}-\Pi_{K}^{2, \nabla} w_{h}\right)^{1 / 2}\right. \\
& \left.\times s_{K}^{\nabla}\left(\widetilde{v}_{h}-\Pi_{K}^{2, \nabla} \widetilde{v}_{h}, \widetilde{v}_{h}-\Pi_{K}^{2, \nabla} \widetilde{v}_{h}\right)^{1 / 2}\right\}+\left\|\left.\Delta w_{h}\right|_{0, \Omega}|| \widetilde{v}-\widetilde{v}_{h}\right\|_{0, \Omega} \\
\leq & \sum_{K \in \mathcal{T}_{h}}\left\{\left|w_{h}\right|_{1, K}|| \Pi_{K}^{1} \nabla \widetilde{v}_{h}-\left.\nabla \widetilde{v}_{h}\right|_{0, K}+c_{3}\left|w_{h}-\Pi_{K}^{2, \nabla} w_{h}\right|_{1, K}\left|\widetilde{v}_{h}-\Pi_{K}^{2, \nabla} \widetilde{v}_{h}\right|_{1, K}\right\} \\
& +\left\|\Delta w_{h}||_{0, \Omega}\right\| \widetilde{v}-\widetilde{v}_{h} \|_{0, \Omega} \\
\leq & \sum_{K \in \mathcal{T}_{h}}\left\{\left|w_{h}\right|_{1, K}|| \boldsymbol{\Pi}_{K}^{1} \nabla \widetilde{v}_{h}-\left.\nabla \widetilde{v}_{h}\right|_{0, K}+c_{3}\left|w_{h}-w_{h}^{\pi}\right|_{1, K}\left|\widetilde{v}_{h}-\widetilde{v}_{h}^{\pi}\right|_{1, K}\right\} \\
& +\left\|\left.\Delta w_{h}\right|_{0, \Omega}\right\| \widetilde{v}-\widetilde{v}_{h} \|_{0, \Omega},
\end{aligned}
$$

for $w_{h}^{\pi}, \widetilde{v}_{h}^{\pi} \in \mathbb{P}_{2}(K)$ such that Proposition 3.2 holds true with respect to $w_{h}$ and $\widetilde{v}_{h}$. Therefore, from Propositions 3.4 and 3.2 , and (4.25), we conclude that

$$
E_{1} \leq C \sum_{K \in \mathcal{T}_{h}}\left\{h_{K}\left|w_{h}\right|_{1, K}\left|\nabla \widetilde{v}_{h}\right|_{1, K}+c_{3} C h_{K}^{2}\left|w_{h}\right|_{2, K}\left|\widetilde{v}_{h}\right|_{2, K}\right\}+C h^{t}|| \Delta w_{h}\left\|_{0, \Omega} \leq C h^{t}\right\| w_{h} \|_{2, \Omega},
$$


with $t \in(1 / 2,1]$ and where we have used in the last inequality the fact that $\left\|\widetilde{v}_{h}\right\|_{2, \Omega} \leq\left\|\widetilde{\mathbf{v}}_{h}\right\|_{2, \Omega}=1$.

Hence, from (4.28), Lemma 4.2 and Theorem 2.1, we get the following estimate (cf. (4.27))

$$
\lambda E_{1}+2 E_{2} \leq C h^{\tilde{s}}\|\mathbf{u}\|_{2+\tilde{s}, \Omega}\left\|\mathbf{w}_{h}\right\|_{2, \Omega} \leq C h^{\tilde{s}}\|f\|_{0, \Omega}\left\|\mathbf{w}_{h}\right\|_{2, \Omega} .
$$

From the above inequality, there exists $h_{0}:=\left(\frac{\bar{c}_{1}}{2 C\|f\|_{0, \Omega}}\right)^{1 / \tilde{s}}>0$ such that for all $h \leq h_{0}$, the following result holds

$$
\sup _{\substack{\mathbf{v}_{h} \in \mathbb{H}_{h} \\\left\|\mathbf{v}_{h}\right\|_{2, \Omega}=1}} A_{h, \mathbf{u}_{I}}\left(\mathbf{w}_{h}, \mathbf{v}_{h}\right) \geq \bar{c}_{1}\left\|\mathbf{w}_{h}\right\|_{2, \Omega}-C h^{\tilde{s}}\|f\|_{0, \Omega}\left\|\mathbf{w}_{h}\right\|_{2, \Omega} \geq \frac{\bar{c}_{1}}{2}\left\|\mathbf{w}_{h}\right\|_{2, \Omega},
$$

where we have used (4.27)-(4.29). The proof is complete.

Now, in what follows, we will focus on proving that the operator $T_{h}$ satisfies the hypotheses of Banach fixedpoint theorem ([22], Thm. 5.7). With this aim, first from Lemma 4.3 we will prove that the operator $T_{h}$ maps the ball

$$
\mathcal{B}\left(\mathbf{u}_{I}, R\right):=\left\{\mathbf{w}_{h} \in \mathbb{H}_{h}:\left\|\mathbf{w}_{h}-\mathbf{u}_{I}\right\|_{2, \Omega} \leq R\right\}
$$

to itself, where $R:=R(h)>0$ is a positive real number depending on $h$ which will be specified later in Lemma 4.5 and we recall that $\mathbf{u}_{I}$ is the interpolant of the isolated solution $\mathbf{u}$. We first need to prove a technical lemma.

Lemma 4.4. Let $\mathbf{w}_{h} \in \mathbb{H}_{h}$. For $h$ small enough, there exists a positive constant $\widetilde{C}$, independent of $h$, such that

$$
\left\|T_{h} \mathbf{w}_{h}-\mathbf{u}_{I}\right\|_{2, \Omega} \leq \widetilde{C}\left(h^{s}+\left\|\mathbf{u}_{I}-\mathbf{w}_{h}\right\|_{2, \Omega}^{2}\right),
$$

where $s \in(1 / 2,1]$ is the Sobolev exponent for the solution of the von Kármán problem (see Thm. 2.1).

Proof. Let $\mathbf{w}_{h} \in \mathbb{H}_{h}$. Since $\left(T_{h} \mathbf{w}_{h}-\mathbf{u}_{I}\right) \in \mathbb{H}_{h}$, we have from Lemma 4.3 (cf. (4.23)) that there exists $\widetilde{c}_{1}>0$ such that

$$
\widetilde{c}_{1}\left\|T \mathbf{w}_{h}-\mathbf{u}_{I}\right\|_{2, \Omega} \leq \sup _{\substack{\mathbf{v}_{h} \in \mathbb{H}_{h} \\\left\|\mathbf{v}_{h}\right\|_{2, \Omega}=1}} A_{h, \mathbf{u}_{I}}\left(T \mathbf{w}_{h}-\mathbf{u}_{I}, \mathbf{v}_{h}\right)
$$

Now, we can choose $\overline{\mathbf{v}}_{h} \in \mathbb{H}_{h} \subseteq \mathbb{H}$ with $\left\|\overline{\mathbf{v}}_{h}\right\|_{2, \Omega}=1$ such that

$$
\widetilde{c}_{1}\left\|T_{h} \mathbf{w}_{h}-\mathbf{u}_{I}\right\|_{2, \Omega} \leq A_{h, \mathbf{u}_{I}}\left(T_{h} \mathbf{w}_{h}-\mathbf{u}_{I}, \overline{\mathbf{v}}_{h}\right) .
$$

Next, using the definitions of $T_{h}$ and $A_{h, \mathbf{u}_{I}}(\cdot, \cdot)$ ( $c f$. (4.3) and (4.2)), and adding the continuous variational formulation (2.12) tested with $\overline{\mathbf{v}}_{h}$ on the right hand side of (4.30), we obtain

$$
\begin{aligned}
& \widetilde{c}_{1}\left\|T_{h} \mathbf{w}_{h}-\mathbf{u}_{I}\right\|_{2, \Omega} \leq A_{h, \mathbf{u}_{I}}\left(T_{h} \mathbf{w}_{h}-\mathbf{u}_{I}, \overline{\mathbf{v}}_{h}\right)=A_{h, \mathbf{u}_{I}}\left(T_{h} \mathbf{w}_{h}, \overline{\mathbf{v}}_{h}\right)-A_{h, \mathbf{u}_{I}\left(\mathbf{u}_{I}, \overline{\mathbf{v}}_{h}\right)} \\
&= 2 B_{h}\left(\mathbf{u}_{I} ; \mathbf{w}_{h}, \overline{\mathbf{v}}_{h}\right)-B_{h}\left(\mathbf{w}_{h} ; \mathbf{w}_{h}, \overline{\mathbf{v}}_{h}\right)+F_{h}\left(\overline{\mathbf{v}}_{h}\right)-A_{h}^{\Delta}\left(\mathbf{u}_{I}, \overline{\mathbf{v}}_{h}\right)-\lambda A_{h}^{\nabla}\left(\mathbf{u}_{I}, \overline{\mathbf{v}}_{h}\right)-2 B_{h}\left(\mathbf{u}_{I} ; \mathbf{u}_{I}, \overline{\mathbf{v}}_{h}\right) \\
&+A^{\Delta}\left(\mathbf{u}, \overline{\mathbf{v}}_{h}\right)+\lambda A^{\nabla}\left(\mathbf{u}, \overline{\mathbf{v}}_{h}\right)+B\left(\mathbf{u} ; \mathbf{u}, \overline{\mathbf{v}}_{h}\right)-F\left(\overline{\mathbf{v}}_{h}\right) \\
&= \underbrace{\left\{2 B_{h}\left(\mathbf{u}_{I} ; \mathbf{w}_{h}, \overline{\mathbf{v}}_{h}\right)-B_{h}\left(\mathbf{w}_{h} ; \mathbf{w}_{h}, \overline{\mathbf{v}}_{h}\right)-B_{h}\left(\mathbf{u}_{I} ; \mathbf{u}_{I}, \overline{\mathbf{v}}_{h}\right)\right\}}_{G_{1}}+\underbrace{\left\{F_{h}\left(\overline{\mathbf{v}}_{h}\right)-F\left(\overline{\mathbf{v}}_{h}\right)\right\}}_{G_{2}} \\
&+\underbrace{\left\{A^{\Delta}\left(\mathbf{u}, \overline{\mathbf{v}}_{h}\right)-A_{h}^{\Delta}\left(\mathbf{u}_{I}, \overline{\mathbf{v}}_{h}\right)\right\}}_{G_{3}}+\underbrace{\left\{B\left(\mathbf{u} ; \mathbf{u}, \overline{\mathbf{v}}_{h}\right)-B_{h}\left(\mathbf{u}_{I} ; \mathbf{u}_{I}, \overline{\mathbf{v}}_{h}\right)\right\}}_{G_{4}\left\{A^{\nabla}\left(\mathbf{u}, \overline{\mathbf{v}}_{h}\right)-A_{h}^{\nabla}\left(\mathbf{u}_{I}, \overline{\mathbf{v}}_{h}\right)\right\}} .
\end{aligned}
$$


In what follows, we want to bound all the terms $G_{i}, i=1, \ldots, 5$ defined in (4.31). Indeed, using the properties of the trilinear form, we rewrite $G_{1}$, then applying the identity (3.16f) and (3.16e) and the fact that $\left\|\overline{\mathbf{v}}_{h}\right\|_{2, \Omega}=1$, we get

$$
\begin{aligned}
G_{1} & =B_{h}\left(\mathbf{u}_{I} ; \mathbf{w}_{h}, \overline{\mathbf{v}}_{h}\right)-B_{h}\left(\mathbf{w}_{h} ; \mathbf{w}_{h}, \overline{\mathbf{v}}_{h}\right)-B_{h}\left(\mathbf{u}_{I} ; \mathbf{u}_{I}, \overline{\mathbf{v}}_{h}\right)+B_{h}\left(\mathbf{u}_{I} ; \mathbf{w}_{h}, \overline{\mathbf{v}}_{h}\right) \\
& =B_{h}\left(\mathbf{u}_{I}-\mathbf{w}_{h} ; \mathbf{w}_{h}, \overline{\mathbf{v}}_{h}\right)+B_{h}\left(\mathbf{u}_{I}-\mathbf{w}_{h} ;-\mathbf{u}_{I}, \overline{\mathbf{v}}_{h}\right) \\
& =B_{h}\left(\mathbf{u}_{I}-\mathbf{w}_{h} ; \mathbf{w}_{h}-\mathbf{u}_{I}, \overline{\mathbf{v}}_{h}\right) \leq C\left\|\mathbf{u}_{I}-\mathbf{w}_{h}\right\|_{2, \Omega}^{2} .
\end{aligned}
$$

For $G_{2}$, we use Cauchy-Schwarz inequality and Lemma 3.4 to obtain

$$
G_{2}=\sum_{K \in \mathcal{T}_{h}} \int_{K} f\left(\Pi_{K}^{2, D} \widetilde{v}_{h}-\widetilde{v}_{h}\right) \leq\|f\|_{0, \Omega}\left\|\Pi_{K}^{2, D} \widetilde{v}_{h}-\widetilde{v}_{h}\right\|_{0, \Omega} \leq C h^{2}\|f\|_{0, \Omega}
$$

Next, to bound $G_{3}$, we use Theorem 2.1 and Proposition 3.2 to find $\mathbf{u}_{\pi} \in\left[\mathbb{P}_{k}(K)\right]^{2}$ such that

$$
\left|\mathbf{u}-\mathbf{u}_{\pi}\right|_{2, K} \leq C h^{s}|\mathbf{u}|_{2+s, K} \quad \forall K \in \mathcal{T}_{h},
$$

with $s \in(1 / 2,1]$.

Now, in the definition of $G_{3}$, we add and subtract the term $\mathbf{u}_{\pi}$, use the definition of $A_{h, K}^{\Delta}(\cdot, \cdot)$, property (3.10), Cauchy-Schwarz inequality, and apply the estimate $(3.12)$ in the definition of $A_{h, K}(\cdot, \cdot)$ to obtain

$$
\begin{aligned}
G_{3} & =\sum_{K \in \mathcal{T}_{h}}\left\{A_{K}^{\Delta}\left(\mathbf{u}-\mathbf{u}_{\pi}, \overline{\mathbf{v}}_{h}\right)+A_{h, K}^{\Delta}\left(\mathbf{u}_{\pi}-\mathbf{u}_{I}, \overline{\mathbf{v}}_{h}\right)\right\} \\
& \leq \sum_{K \in \mathcal{T}_{h}}\left\{\left|\mathbf{u}-\mathbf{u}_{\pi}\right|_{2, K}\left|\overline{\mathbf{v}}_{h}\right|_{2, K}+A_{h, K}^{\Delta}\left(\mathbf{u}_{\pi}-\mathbf{u}_{I}, \mathbf{u}_{\pi}-\mathbf{u}_{I}\right)^{1 / 2} A_{h, K}^{\Delta}\left(\overline{\mathbf{v}}_{h}, \overline{\mathbf{v}}_{h}\right)^{1 / 2}\right\} \\
& \leq \sum_{K \in \mathcal{T}_{h}}\left\{\left|\mathbf{u}-\mathbf{u}_{\pi}\right|_{2, K}\left|\overline{\mathbf{v}}_{h}\right|_{2, K}+\alpha_{2}\left|\mathbf{u}_{\pi}-\mathbf{u}_{I}\right|_{2, K}\left|\overline{\mathbf{v}}_{h}\right|_{2, K}\right\} .
\end{aligned}
$$

Next, adding and subtracting the term $\mathbf{u}$, using the estimates (4.34) and (4.1), and Theorem 2.1 on the right hand side of the above term, we get

$$
\begin{aligned}
G_{3} & \leq \sum_{K \in \mathcal{T}_{h}}\left\{\left(1+\alpha_{2}\right)\left|\mathbf{u}-\mathbf{u}_{\pi}\right|_{2, K}\left|\overline{\mathbf{v}}_{h}\right|_{2, K}+\alpha_{2}\left|\mathbf{u}-\mathbf{u}_{I}\right|_{2, K}\left|\overline{\mathbf{v}}_{h}\right|_{2, K}\right\} \\
& \leq C h^{s}\|\mathbf{u}\|_{2+s, \Omega}\left\|\overline{\mathbf{v}}_{h}\right\|_{2, \Omega}=C h^{s}\|\mathbf{u}\|_{2+s, \Omega} \leq C h^{s}\|f\|_{0, \Omega} .
\end{aligned}
$$

For the term $G_{4}$, we use the definition of $\boldsymbol{\Pi}_{K}^{1}$, Cauchy Schwarz inequality, and (3.9) to obtain

$$
\begin{aligned}
G_{4} & =\sum_{K \in \mathcal{T}_{h}}\left\{\int_{K}\left\{\boldsymbol{\Pi}_{K}^{1} \nabla u_{I}-\nabla u\right\} \cdot \nabla \widetilde{v}_{h}+s_{K}^{\nabla}\left(\Pi_{K}^{2, \nabla} u_{I}-u_{I}, \widetilde{v}_{h}-\Pi_{K}^{2, \nabla} \widetilde{v}_{h}\right)\right\} \\
& \leq \sum_{K \in \mathcal{T}_{h}}\left\{|| \Pi_{K}^{1} \nabla u_{I}-\left.\nabla u\right|_{0, K}\left|\widetilde{v}_{h}\right|_{1, K}+c_{3}\left|u_{I}-\Pi_{K}^{2, \nabla} u_{I}\right|_{1, K}\left|\widetilde{v}_{h}-\Pi_{K}^{2, \nabla} \widetilde{v}_{h}\right|_{1, K}\right\} \\
& \leq \sum_{K \in \mathcal{T}_{h}}\left\{\left(|| \Pi_{K}^{1} \nabla u_{I}-\nabla u_{I}||_{0, K}+\|\left.\nabla\left(u-u_{I}\right)\right|_{0, K}\right)\left|\widetilde{v}_{h}\right|_{1, K}+c_{3}\left|u_{I}-\Pi_{K}^{2, \nabla} u_{I}\right|_{1, K}\left|\widetilde{v}_{h}-\Pi_{K}^{2, \nabla} \widetilde{v}_{h}\right|_{1, K}\right\},
\end{aligned}
$$

where in the last step we have added and subtracted $\nabla u_{I}$.

Now, on the right hand side above, we apply Propositions 3.4 and 3.3, the definition of $\Pi_{K}^{2, \nabla}$, Proposition 3.2 and finally Theorem 2.1, to deduce that

$$
\begin{aligned}
G_{4} & \leq C \sum_{K \in \mathcal{T}_{h}}\left\{\left\{h_{K}\left|u_{I}\right|_{2, K}+h_{K}^{1+s}|u|_{2, K}\right\}\left|\widetilde{v}_{h}\right|_{1, K}+h_{K}^{2}\left|u_{I}\right|_{2, K}\left|\widetilde{v}_{h}\right|_{2, K}\right\} \\
& \leq C h\|\mathbf{u}\|_{2+s, \Omega}\left\|\overline{\mathbf{v}}_{h}\right\|_{2, \Omega}=C h\|\mathbf{u}\|_{2+s, \Omega} \leq C h\|f\|_{0, \Omega} .
\end{aligned}
$$


It is easy to check that $G_{5}$ can be bounded using the same arguments as those applied to estimate (4.15). Hence, we obtain the following result

$$
G_{5} \leq C h^{s}\|\mathbf{u}\|_{2+s, \Omega}\left\|\overline{\mathbf{v}}_{h}\right\|_{2, \Omega}=C h^{s}\|\mathbf{u}\|_{2+s, \Omega} \leq C h^{s}\|f\|_{0, \Omega} .
$$

Therefore, by combining (4.31) and the estimates (4.32), (4.33), (4.35)-(4.37), we obtain that

$$
\left\|T_{h} \mathbf{w}_{h}-\mathbf{u}_{I}\right\|_{2, \Omega} \leq \widetilde{C}\left(h^{s}+\left\|\mathbf{u}_{I}-\mathbf{w}_{h}\right\|_{2, \Omega}^{2}\right),
$$

where $\widetilde{C}$ is a positive constant depending on $\|f\|_{0, \Omega}$, and $s \in(1 / 2,1]$ is the Sobolev exponent for the solution of the von Kármán problem (see Thm. 2.1).

Now, we are in position to prove that $T_{h}$ maps the ball $\mathcal{B}\left(\mathbf{u}_{I}, R\right)$ to itself.

Lemma 4.5. For $h$ small enough, there exists a positive constant $R(h)$, depending on $h$, such that

$$
T_{h}\left(\mathcal{B}\left(\mathbf{u}_{I}, R(h)\right)\right) \subseteq \mathcal{B}\left(\mathbf{u}_{I}, R(h)\right) .
$$

Proof. Let $\mathbf{z}_{h} \in T_{h}\left(\mathcal{B}\left(\mathbf{u}_{I}, R\right)\right)$, then there exists $\widetilde{\mathbf{w}}_{h} \in \mathbb{H}_{h}$ such that $\mathbf{z}_{h}=T_{h} \widetilde{\mathbf{w}}_{h}$ and $\left\|\widetilde{\mathbf{w}}_{h}-\mathbf{u}_{I}\right\|_{2, \Omega} \leq R$. Then, applying Lemma 4.4, we have

$$
\left\|\mathbf{z}_{h}-\mathbf{u}_{I}\right\|_{2, \Omega}=\left\|T_{h} \widetilde{\mathbf{w}}_{h}-\mathbf{u}_{I}\right\|_{2, \Omega} \leq \widetilde{C}\left(h^{s}+\left\|\mathbf{u}_{I}-\widetilde{\mathbf{w}}_{h}\right\|_{2, \Omega}^{2}\right)
$$

Now, for all $h \leq h_{1}:=(2 \widetilde{C})^{-2 / s}$, we choose $2 \widetilde{C} h^{s}=: R(h)=R$ and obtain

$$
\left\|\mathbf{z}_{h}-\mathbf{u}_{I}\right\|_{2, \Omega} \leq \widetilde{C} h^{s}+\widetilde{C}\left\|\widetilde{\mathbf{w}}_{h}-\mathbf{u}_{I}\right\|_{2, \Omega}^{2} \leq \widetilde{C} h^{s}+\widetilde{C} R(h)^{2}=\frac{R(h)}{2}+\frac{R(h)}{2} 4 \widetilde{C}^{2} h^{s} \leq R(h) .
$$

Therefore $\mathbf{z}_{h} \in \mathcal{B}\left(\mathbf{u}_{I}, R(h)\right)$.

In the following result, we will prove that the operator $T_{h}$ is a contraction in $\mathcal{B}\left(\mathbf{u}_{I}, R(h)\right)$.

Lemma 4.6. For $h$ small enough, the operator $T_{h}$ is a contraction in $\mathcal{B}\left(\mathbf{u}_{I}, R(h)\right)$.

Proof. Let $\mathbf{w}_{h}, \widetilde{\mathbf{w}}_{h} \in \mathcal{B}\left(\mathbf{u}_{I}, R(h)\right)$, hence

$$
\left\|\mathbf{w}_{h}-\mathbf{u}_{I}\right\|_{2, \Omega},\left\|\widetilde{\mathbf{w}}_{h}-\mathbf{u}_{I}\right\|_{2, \Omega} \leq R(h)=2 \widetilde{C} h^{s} .
$$

Now, from the definition of operator $T_{h}(c f .(4.3))$, we have

$$
\begin{array}{ll}
A_{h, \mathbf{u}_{I}}\left(T_{h} \mathbf{w}_{h}, \mathbf{v}_{h}\right)=2 B_{h}\left(\mathbf{u}_{I} ; \mathbf{w}_{h}, \mathbf{v}_{h}\right)-B_{h}\left(\mathbf{w}_{h} ; \mathbf{w}_{h}, \mathbf{v}_{h}\right)+F_{h}\left(\mathbf{v}_{h}\right) & \forall \mathbf{v}_{h} \in \mathbb{H}_{h} ; \\
A_{h, \mathbf{u}_{I}}\left(T_{h} \widetilde{\mathbf{w}}_{h}, \mathbf{v}_{h}\right)=2 B_{h}\left(\mathbf{u}_{I} ; \widetilde{\mathbf{w}}_{h}, \mathbf{v}_{h}\right)-B_{h}\left(\widetilde{\mathbf{w}}_{h} ; \widetilde{\mathbf{w}}_{h}, \mathbf{v}_{h}\right)+F_{h}\left(\mathbf{v}_{h}\right) & \forall \mathbf{v}_{h} \in \mathbb{H}_{h},
\end{array}
$$

which implies

$$
A_{h, \mathbf{u}_{I}}\left(T_{h}\left(\mathbf{w}_{h}-\widetilde{\mathbf{w}}_{h}\right), \mathbf{v}_{h}\right)=2 B_{h}\left(\mathbf{u}_{I} ; \mathbf{w}_{h}-\widetilde{\mathbf{w}}_{h}, \mathbf{v}_{h}\right)-B_{h}\left(\mathbf{w}_{h} ; \mathbf{w}_{h}, \mathbf{v}_{h}\right)+B_{h}\left(\widetilde{\mathbf{w}}_{h} ; \widetilde{\mathbf{w}}_{h}, \mathbf{v}_{h}\right) \quad \forall \mathbf{v}_{h} \in \mathbb{H}_{h} .
$$

Using Lemma 4.3 , we can choose $\overline{\mathbf{v}}_{h} \in \mathbb{H}_{h}$ with $\left\|\overline{\mathbf{v}}_{h}\right\|_{2, \Omega}=1$ such that

$$
C\left\|T_{h} \mathbf{w}_{h}-T_{h} \widetilde{\mathbf{w}}_{h}\right\|_{2, \Omega}=C\left\|T_{h}\left(\mathbf{w}_{h}-\widetilde{\mathbf{w}}_{h}\right)\right\|_{2, \Omega} \leq A_{h, \mathbf{u}_{I}}\left(T_{h}\left(\mathbf{w}_{h}-\widetilde{\mathbf{w}}_{h}\right), \overline{\mathbf{v}}_{h}\right) .
$$


Therefore, by combining (4.39) and (4.40), using (3.16e) and (3.16f), we get

$$
\begin{aligned}
\left\|T_{h} \mathbf{w}_{h}-T_{h} \widetilde{\mathbf{w}}_{h}\right\|_{2, \Omega} & \leq \hat{C}\left\{2 B_{h}\left(\mathbf{u}_{I} ; \mathbf{w}_{h}-\widetilde{\mathbf{w}}_{h}, \overline{\mathbf{v}}_{h}\right)-B_{h}\left(\mathbf{w}_{h} ; \mathbf{w}_{h}, \overline{\mathbf{v}}_{h}\right)+B_{h}\left(\widetilde{\mathbf{w}}_{h} ; \widetilde{\mathbf{w}}_{h}, \overline{\mathbf{v}}_{h}\right)\right\} \\
& =\hat{C}\left\{B_{h}\left(\widetilde{\mathbf{w}}_{h}-\mathbf{w}_{h} ; \mathbf{w}_{h}-\mathbf{u}_{I}, \overline{\mathbf{v}}_{h}\right)+B_{h}\left(\widetilde{\mathbf{w}}_{h}-\mathbf{u}_{I} ; \widetilde{\mathbf{w}}_{h}-\mathbf{w}_{h}, \overline{\mathbf{v}}_{h}\right)\right\} \\
& \leq \bar{C}\left\{\left\|\widetilde{\mathbf{w}}_{h}-\mathbf{w}_{h}\right\|_{2, \Omega}\left\|\mathbf{w}_{h}-\mathbf{u}_{I}\right\|_{2, \Omega}\left\|\overline{\mathbf{v}}_{h}\right\|_{2, \Omega}+\left\|\widetilde{\mathbf{w}}_{h}-\mathbf{w}_{h}\right\|_{2, \Omega}\left\|\widetilde{\mathbf{w}}_{h}-\mathbf{u}_{I}\right\|_{2, \Omega}\left\|\overline{\mathbf{v}}_{h}\right\|_{2, \Omega}\right\} \\
& =\bar{C}\left\|\widetilde{\mathbf{w}}_{h}-\mathbf{w}_{h}\right\|_{2, \Omega}\left\{\left\|\mathbf{w}_{h}-\mathbf{u}_{I}\right\|_{2, \Omega}+\left\|\widetilde{\mathbf{w}}_{h}-\mathbf{u}_{I}\right\|_{2, \Omega}\right\} .
\end{aligned}
$$

Finally, for all $h \leq h_{2}:=(8 \bar{C} \widetilde{C})^{-1 / s}$, we apply (4.38) on the right hand side of (4.41) to obtain

$$
\left\|T_{h} \mathbf{w}_{h}-T_{h} \widetilde{\mathbf{w}}_{h}\right\|_{2, \Omega} \leq 2 \bar{C} R(h)\left\|\mathbf{w}_{h}-\widetilde{\mathbf{w}}_{h}\right\|_{2, \Omega}=4 \bar{C} \widetilde{C} h^{s}\left\|\mathbf{w}_{h}-\widetilde{\mathbf{w}}_{h}\right\|_{2, \Omega} \leq \frac{1}{2}\left\|\mathbf{w}_{h}-\widetilde{\mathbf{w}}_{h}\right\|_{2, \Omega} .
$$

Therefore, we have finished the proof.

Finally, we are ready to prove that the discrete problem (3.15) admits a unique solution.

Theorem 4.1. Let $\mathbf{u}$ be an isolated solution of (2.12). Then, for $h$ small enough, the discrete problem (3.15) has a unique solution $\mathbf{u}_{h} \in \mathbb{H}_{h}$. Moreover, we have that

$$
\left\|\mathbf{u}_{h}-\mathbf{u}_{I}\right\|_{2, \Omega} \leq C h^{s}
$$

Proof. We know that the solution of (3.15) is a fixed point of operator $T_{h}$. Thus, the proof follows from Lemmas 4.5 and 4.6, and Banach fixed-point theorem.

We finish this section presenting the following result, which provides the rate of convergence of our virtual element scheme.

Theorem 4.2. Let $\mathbf{u}$ and $\mathbf{u}_{h}$ be the isolated solution of (2.12) and the unique solution of the discrete problem (3.15), respectively. Then, there exists a positive constant $C$, that depends on the force function $f$ but independent of mesh size $h$, such that for all $h \leq \min \left\{h_{1}, h_{2}\right\}$ we have that

$$
\left\|\mathbf{u}-\mathbf{u}_{h}\right\|_{2, \Omega} \leq C h^{s},
$$

where $s \in(1 / 2,1]$ is the Sobolev exponent for the solution of von Kármán problem (see Thm. 2.1).

Proof. For all $h \leq \min \left\{h_{1}, h_{2}\right\}$, we have from Theorem 4.1 the following estimate

$$
\left\|\mathbf{u}_{h}-\mathbf{u}_{I}\right\|_{2, \Omega} \leq C h^{s}
$$

Hence, applying triangle inequality in the term $\left\|\mathbf{u}-\mathbf{u}_{h}\right\|_{2, \Omega}$, using the estimates (4.1), (4.42), and Theorem 2.1, we deduce

$$
\begin{aligned}
\left\|\mathbf{u}-\mathbf{u}_{h}\right\|_{2, \Omega} & \leq\left\|\mathbf{u}-\mathbf{u}_{I}\right\|_{2, \Omega}+\left\|\mathbf{u}_{I}-\mathbf{u}_{h}\right\|_{2, \Omega} \\
& \leq C h^{s}\|\mathbf{u}\|_{2+s, \Omega}+\left\|\mathbf{u}_{I}-\mathbf{u}_{h}\right\|_{2, \Omega} \\
& \leq C h^{s}
\end{aligned}
$$

where $C$ is a positive constant depending on $\|f\|_{0, \Omega}$. The proof is complete. 

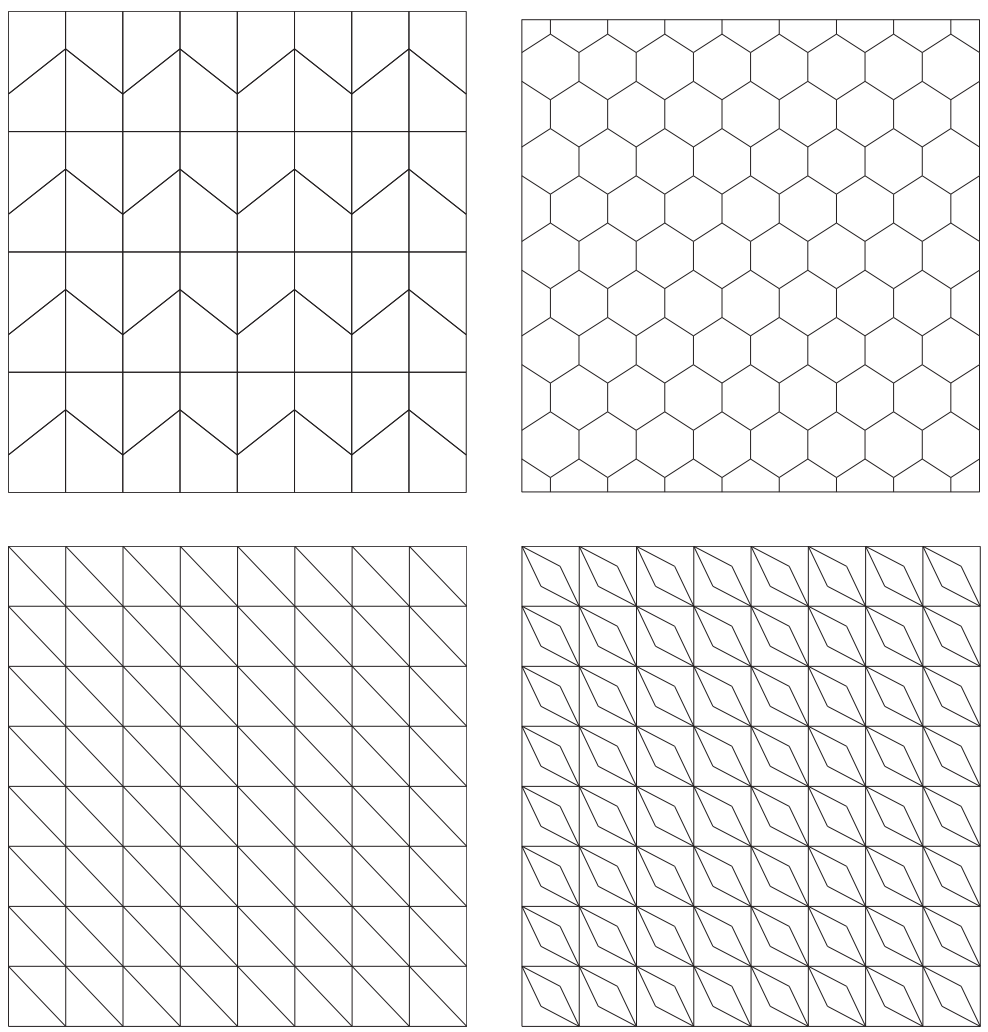

Figure 2. Sample meshes: $\mathcal{T}_{h}^{1}$ (top left), $\mathcal{T}_{h}^{2}$ (top right), $\mathcal{T}_{h}^{3}$ (bottom left) and $\mathcal{T}_{h}^{4}$ (bottom right).

\section{Numerical ReSults}

We report in this section a series of numerical experiments to approximate the isolated solutions of the von Kármán problem (2.12), using the Virtual Element Method proposed and analyzed in this paper. We have implemented in a MATLAB code the proposed VEM on arbitrary polygonal meshes (see [10]).

We will test the method by using different families of meshes (see Fig. 2):

- $\mathcal{T}_{h}^{1}$ : trapezoidal meshes which consist of partitions of the domain into $N \times N$ congruent trapezoids, all similar to the trapezoid with vertices $(0,0),(1 / 2,0),(1 / 2,2 / 3)$ and $(0,1 / 3)$;

$-\mathcal{T}_{h}^{2}$ : hexagonal meshes;

$-\mathcal{T}_{h}^{3}$ : triangular meshes;

$-\mathcal{T}_{h}^{4}$ : distorted concave rhombic quadrilaterals.

Now, in order to complete the choice of the VEM, we have to fix the bilinear forms $s_{K}^{D}(\cdot, \cdot)$ and $s_{K}^{\nabla}(\cdot, \cdot)$ satisfying (3.8) and (3.9), respectively. First, we consider the following symmetric bilinear forms (see for instance $[8,45]):$

$$
\begin{array}{ll}
s_{K}^{D}\left(u_{h}, v_{h}\right):=\sigma_{K} \sum_{i=1}^{N_{K}}\left[u_{h}\left(\mathrm{v}_{i}\right) v_{h}\left(\mathrm{v}_{i}\right)+h_{\mathrm{v}_{i}}^{2} \nabla u_{h}\left(\mathrm{v}_{i}\right) \cdot \nabla v_{h}\left(\mathrm{v}_{i}\right)\right] & \forall u_{h}, v_{h} \in H_{h}^{K}, \\
s_{K}^{\nabla}\left(u_{h}, v_{h}\right):=\bar{\sigma}_{K} \sum_{i=1}^{N_{K}}\left[u_{h}\left(\mathrm{v}_{i}\right) v_{h}\left(\mathrm{v}_{i}\right)+h_{\mathrm{v}_{i}}^{2} \nabla u_{h}\left(\mathrm{v}_{i}\right) \cdot \nabla v_{h}\left(\mathrm{v}_{i}\right)\right] & \forall u_{h}, v_{h} \in H_{h}^{K},
\end{array}
$$


TABLE 1. Test 1: Errors and experimental convergence rates $\mathrm{e}_{0}\left(u_{h}\right), \mathrm{e}_{1}\left(u_{h}\right), \mathrm{e}_{2}\left(u_{h}\right), \mathrm{e}_{0}\left(\psi_{h}\right)$, $\mathrm{e}_{1}\left(\psi_{h}\right)$ and $\mathrm{e}_{2}\left(\psi_{h}\right)$ of the discrete solution to the von Kármán problem.

\begin{tabular}{|c|c|c|c|c|c|c|c|}
\hline $\mathcal{T}_{h}$ & $N_{\text {dof }}$ & $\mathrm{e}_{0}\left(u_{h}\right)$ & $\operatorname{rc}\left(u_{h}\right)$ & $\mathrm{e}_{1}\left(u_{h}\right)$ & $\operatorname{rc}\left(u_{h}\right)$ & $\mathrm{e}_{2}\left(u_{h}\right)$ & $\operatorname{rc}\left(u_{h}\right)$ \\
\hline \multirow{5}{*}{$\mathcal{T}_{h}^{3}$} & 54 & 0.000431704768438 & - & 0.003262724422857 & - & 0.031728922182966 & - \\
\hline & 294 & 0.000118318604331 & 1.87 & 0.000935470949060 & 1.80 & 0.016255461918515 & 0.96 \\
\hline & 1350 & 0.000029867487367 & 1.99 & 0.000239063199567 & 1.97 & 0.008150175184542 & 1.00 \\
\hline & 5766 & 0.000007461004475 & 2.00 & 0.000059929912000 & 2.00 & 0.004079547948378 & 1.00 \\
\hline & 23814 & 0.000001863069256 & 2.00 & 0.000014979870677 & 2.00 & 0.002040881649304 & 1.00 \\
\hline \multirow{5}{*}{$\mathcal{T}_{h}^{4}$} & 246 & 0.000480588388535 & - & 0.003317993901837 & - & 0.031341253980477 & - \\
\hline & 1062 & 0.000136460315064 & 1.72 & 0.000988414232088 & 1.66 & 0.015733875049155 & 0.94 \\
\hline & 4422 & 0.000034563080364 & 1.93 & 0.000256091744078 & 1.89 & 0.007800044026566 & 0.98 \\
\hline & 18054 & 0.000008594114090 & 1.98 & 0.000064363787275 & 1.96 & 0.003890338441267 & 0.99 \\
\hline & 72966 & 0.000002136931492 & 1.99 & 0.000016086825337 & 1.99 & 0.001944197375647 & 0.99 \\
\hline $\mathcal{T}_{h}$ & $N_{\text {dof }}$ & $\mathrm{e}_{0}\left(\psi_{h}\right)$ & $\operatorname{rc}\left(\psi_{h}\right)$ & $\mathrm{e}_{1}\left(\psi_{h}\right)$ & $\operatorname{rc}\left(\psi_{h}\right)$ & $\mathrm{e}_{2}\left(\psi_{h}\right)$ & $\mathrm{rc}\left(\psi_{h}\right)$ \\
\hline \multirow{5}{*}{$\mathcal{T}_{h}^{1}$} & 54 & 0.073050506944122 & - & 0.846040099702635 & - & 8.159441640704209 & - \\
\hline & 294 & 0.012011400077328 & 2.60 & 0.292568564494940 & 1.53 & 4.341739275488559 & 0.91 \\
\hline & 1350 & 0.002294277174721 & 2.39 & 0.083090515350743 & 1.81 & 2.174289254445207 & 1.00 \\
\hline & 5766 & 0.000499349745906 & 2.20 & 0.021622526054148 & 1.94 & 1.083299492719920 & 1.01 \\
\hline & 23814 & 0.000119140487292 & 2.08 & 0.005465045349110 & 1.98 & 0.540966031106735 & 1.00 \\
\hline \multirow{5}{*}{$\mathcal{T}_{h}^{2}$} & 192 & 0.061884511675239 & - & 0.679874909970360 & - & 7.512913582695741 & - \\
\hline & 768 & 0.026172828966453 & 1.46 & 0.249463389030945 & 1.71 & 4.077763689322426 & 1.04 \\
\hline & 3072 & 0.008664638440498 & 1.74 & 0.071485859700407 & 1.97 & 2.055397431297525 & 1.08 \\
\hline & 12288 & 0.002491920671679 & 1.88 & 0.018172983843658 & 2.06 & 1.024040359048393 & 1.05 \\
\hline & 49152 & 0.000669515058352 & 1.94 & 0.004531873249613 & 2.05 & 0.510867678265516 & 1.03 \\
\hline
\end{tabular}

where $\mathrm{v}_{1}, \ldots, \mathrm{v}_{N_{K}}$ denote the vertices of $K, h_{\mathrm{v}_{i}}$ is chosen as the maximum diameter of the elements $K$ with $\mathrm{v}_{i}$ as a vertex. Moreover, $\sigma_{K}$ and $\bar{\sigma}_{K}$ are multiplicative factors to consider the $h$-scaling and the physical constants of the problem. For instance, in the numerical tests, we have considered $\sigma_{K}, \bar{\sigma}_{K}>0$ as the mean value of the eigenvalues of the local matrices $a_{K}^{\Delta}\left(\Pi_{K}^{2, D} \phi_{i}, \Pi_{K}^{2, D} \phi_{j}\right)$ and $a_{K}^{\nabla}\left(\Pi_{K}^{2, \nabla} \phi_{i}, \Pi_{K}^{2, \nabla} \phi_{j}\right)$, respectively, where $\left\{\phi_{i}\right\}_{i=1}^{\operatorname{dim}} H_{h}^{K}$ corresponds to a basis of $H_{h}^{K}$.

In order to present the numerical tests, we have taken as computational domain $\Omega:=(0,1)^{2}$. The discrete solution associated to problem (3.15) was obtained by a classical Newton method. In particular, we have considered the usual incremental loading procedure (see for instance [11], Sect. 3.2) to approximate the discrete solution of the nonlinear von Kármán problem: given a positive integer $\hat{N}$, let $F_{h}^{\ell}\left(\mathbf{v}_{h}\right)=(\ell / \hat{N}) F_{h}\left(\mathbf{v}_{h}\right) \forall \ell=1,2, \ldots, \hat{N}$ be the partial loadings. Therefore, given the initial guest $\mathbf{u}_{h}^{0}$ (for instance, the zero function), one applies for $\ell=1,2, \ldots, \hat{N}$ the following iterative procedure

Given $\mathbf{u}_{h}^{0}$ do

$$
\begin{aligned}
& \text { for } \ell=1: \hat{N} \text { do } \\
& \begin{array}{l}
\quad F_{h}^{\ell}\left(\mathbf{v}_{h}\right)=(\ell / \hat{N}) F_{h}\left(\mathbf{v}_{h}\right) \\
\quad \text { Solve Newton iterates } \\
A_{h}^{\Delta}\left(\mathbf{u}_{h}^{\ell}, \mathbf{v}_{h}\right)+\lambda A_{h}^{\nabla}\left(\mathbf{u}_{h}^{\ell}, \mathbf{v}_{h}\right)+2 B_{h}\left(\mathbf{u}_{h}^{\ell-1} ; \mathbf{v}_{h}^{\ell}, \mathbf{v}_{h}\right)=B_{h}\left(\mathbf{u}_{h}^{\ell-1} ; \mathbf{u}_{h}^{\ell-1}, \mathbf{v}_{h}\right)+F_{h}^{\ell}\left(\mathbf{v}_{h}\right)
\end{array} \\
& \text { end }
\end{aligned}
$$

Thus, the final solution is $\mathbf{u}_{h}:=\mathbf{u}_{h}^{\hat{N}}$.

Moreover, we define the individual errors by:

$$
\mathrm{e}_{0}\left(w_{h}\right):=|| w-\Pi_{h}^{2, D} w_{h} \|_{0, \Omega}, \quad \mathrm{e}_{1}\left(w_{h}\right):=\left|w-\Pi_{h}^{2, D} w_{h}\right|_{1, h}, \quad \mathrm{e}_{2}\left(w_{h}\right):=\left|w-\Pi_{h}^{2, D} w_{h}\right|_{2, h},
$$



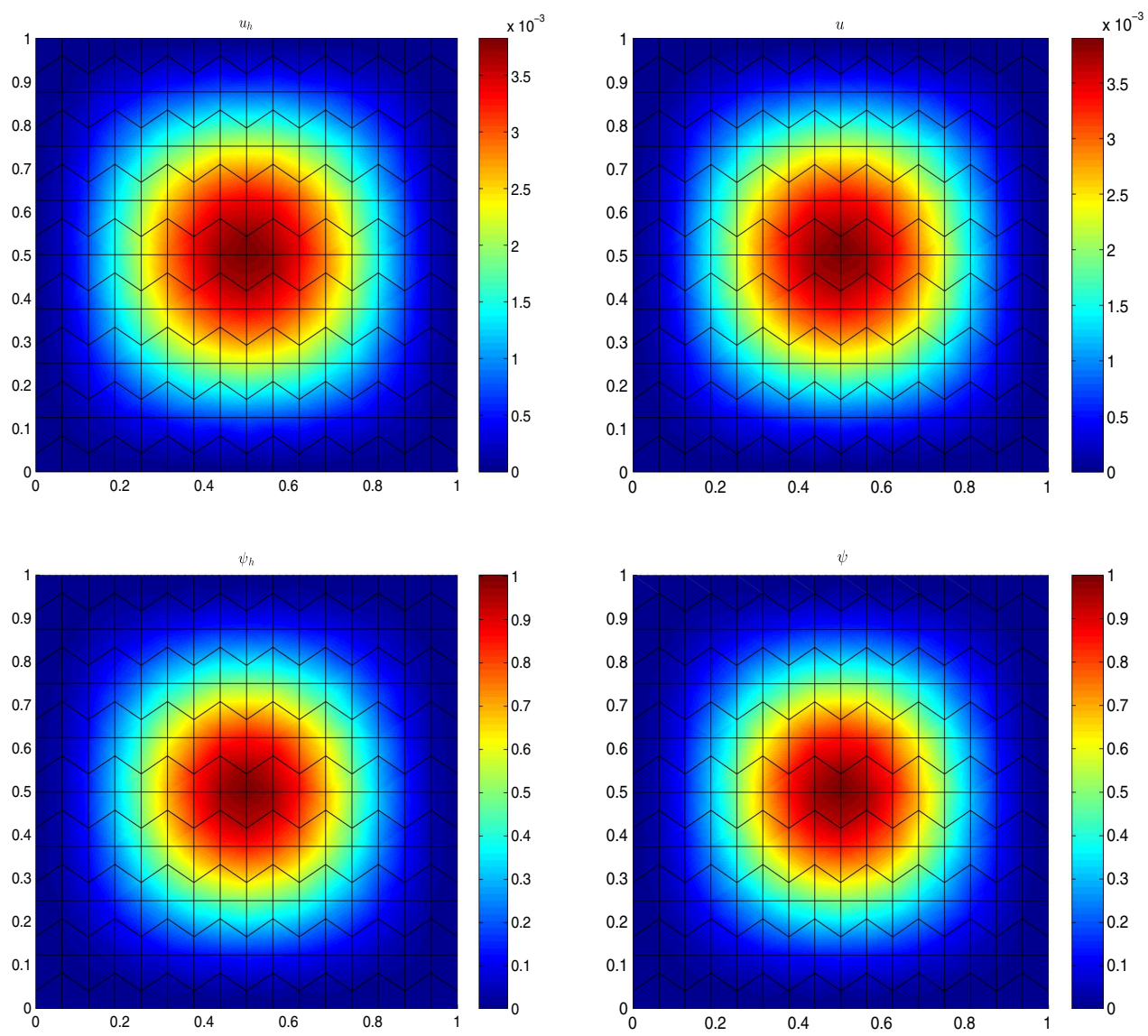

FiguRE 3. Test 1. $u_{h}$ (top left), $u$ (top right), $\psi_{h}$ (bottom left) and $\psi$ (bottom right).

where $\Pi_{h}^{2, D}$ has been defined in (3.17).

We have computed experimental rates of convergence for each individual error as follows:

$$
\mathrm{rc}(\cdot):=\frac{\log \left(\mathrm{e}_{\star}(\cdot) / \mathrm{e}_{\star}^{\prime}(\cdot)\right)}{\log \left(N_{\mathrm{dof}} / N_{\mathrm{dof}}^{\prime}\right)} \quad \text { for all subscripts } \star \in\{0,1,2\},
$$

with $N_{\text {dof }}$ and $N_{\text {dof }}^{\prime}$ denote the degrees of freedom of two consecutive polygonal decompositions with respectively errors $\mathrm{e}_{\star}$ and $\mathrm{e}_{\star}^{\prime}$. For each mesh $\mathcal{T}_{h}$, the degrees of freedom are $N_{\text {dof }}=6 N_{v}$, where $N_{v}$ denotes the number of interior vertices of the polygonal mesh.

In what follows, we present three numerical tests illustrating the performance of our virtual element scheme. For reasons of brevity, we do not report the results obtained with all meshes for all test problems. However, all non reported results are in accordance with the ones shown.

\subsection{Test 1}

In this test, we consider the following variation of system (2.4), where we have modified the right hand side of the second equation. 
TABLE 2. Test 2: Errors and experimental convergence rates $\mathrm{e}_{0}\left(u_{h}\right), \mathrm{e}_{1}\left(u_{h}\right), \mathrm{e}_{2}\left(u_{h}\right), \mathrm{e}_{0}\left(\psi_{h}\right)$, $\mathrm{e}_{1}\left(\psi_{h}\right)$ and $\mathrm{e}_{2}\left(\psi_{h}\right)$ of the discrete solution to the von Kármán problem.

\begin{tabular}{|c|c|c|c|c|c|c|c|}
\hline $\mathcal{T}_{h}$ & $N_{\text {dof }}$ & $\mathrm{e}_{0}\left(u_{h}\right)$ & $\operatorname{rc}\left(u_{h}\right)$ & $\mathrm{e}_{1}\left(u_{h}\right)$ & $\operatorname{rc}\left(u_{h}\right)$ & $\mathrm{e}_{2}\left(u_{h}\right)$ & $\operatorname{rc}\left(u_{h}\right)$ \\
\hline \multirow{5}{*}{$\mathcal{T}_{h}^{1}$} & 54 & 0.003331813500703 & - & 0.040541588728628 & - & 0.392326399686893 & - \\
\hline & 294 & 0.000666755182746 & 1.90 & 0.013573656090400 & 1.29 & 0.211617144042376 & 0.73 \\
\hline & 1350 & 0.000139272569159 & 2.05 & 0.003744148938304 & 1.69 & 0.107073487968784 & 0.89 \\
\hline & 5766 & 0.000032059141547 & 2.02 & 0.000964443541553 & 1.87 & 0.053613559930229 & 0.95 \\
\hline & 23814 & 0.000007817172339 & 1.99 & 0.000243151609540 & 1.94 & 0.026821738028166 & 0.98 \\
\hline \multirow{5}{*}{$\mathcal{T}_{h}^{2}$} & 192 & 0.003338044048468 & - & 0.029905766623389 & - & 0.331352206962454 & - \\
\hline & 768 & 0.001214026245280 & 1.46 & 0.011071959441869 & 1.43 & 0.188066935651525 & 0.82 \\
\hline & 3072 & 0.000374019547265 & 1.70 & 0.003255655646633 & 1.77 & 0.098467208145884 & 0.93 \\
\hline & 12288 & 0.000107769516445 & 1.80 & 0.000862676994761 & 1.92 & 0.050139745754829 & 0.97 \\
\hline & 49152 & 0.000029635952218 & 1.86 & 0.000221290022671 & 1.96 & 0.025284764141485 & 0.99 \\
\hline $\mathcal{T}_{h}$ & $N_{\text {dof }}$ & $\mathrm{e}_{0}\left(\psi_{h}\right)$ & $\operatorname{rc}\left(\psi_{h}\right)$ & $\mathrm{e}_{1}\left(\psi_{h}\right)$ & $\operatorname{rc}\left(\psi_{h}\right)$ & $\mathrm{e}_{2}\left(\psi_{h}\right)$ & $\operatorname{rc}\left(\psi_{h}\right)$ \\
\hline \multirow{5}{*}{$\mathcal{T}_{h}^{3}$} & 54 & 0.029494648554614 & - & 0.412210903728138 & - & 6.003264759584535 & - \\
\hline & 294 & 0.004591865781598 & 2.20 & 0.101028788029534 & 1.66 & 3.069362969416609 & 0.79 \\
\hline & 1350 & 0.000940086309599 & 2.08 & 0.025008571679680 & 1.83 & 1.540413925828407 & 0.90 \\
\hline & 5766 & 0.000222681115380 & 1.98 & 0.006215447146812 & 1.92 & 0.770497464565608 & 0.95 \\
\hline & 23814 & 0.000055123897439 & 1.97 & 0.001548657099701 & 1.96 & 0.385190062299381 & 0.98 \\
\hline \multirow{5}{*}{$\mathcal{T}_{h}^{4}$} & 246 & 0.027814402349946 & - & 0.372966458416872 & - & 5.590747627283569 & - \\
\hline & 1062 & 0.004522053574370 & 2.62 & 0.094464604014345 & 1.98 & 2.885848302050357 & 0.95 \\
\hline & 4422 & 0.000957131357883 & 2.24 & 0.023604212432399 & 2.00 & 1.453857116146261 & 0.99 \\
\hline & 18054 & 0.000229063918352 & 2.06 & 0.005877183318359 & 2.01 & 0.728469592800096 & 1.00 \\
\hline & 72966 & 0.000056858787426 & 2.01 & 0.001464852130236 & 2.00 & 0.364490348651261 & 1.00 \\
\hline
\end{tabular}

$$
\left\{\begin{array}{rlr}
\Delta^{2} u+\lambda \Delta u-[\psi, u] & =f & \text { in } \Omega, \\
\Delta^{2} \psi+\frac{1}{2}[u, u] & =g & \text { in } \Omega, \\
u=\partial_{\nu} u=0 & \text { on } \Gamma, \\
\psi=\partial_{\nu} \psi=0 & \text { on } \Gamma .
\end{array}\right.
$$

We consider $\lambda=5$ and the right hand side functions $f$ and $g$ so that the exact solution $(u, \psi)$ of $(5.1)$ is given by

$$
u(x, y)=x^{2} y^{2}(x-1)^{2}(y-1)^{2} \quad \text { in } \Omega, \quad \text { and } \quad \psi(x, y)=\sin ^{2}(\pi x) \sin ^{2}(\pi y) \quad \text { in } \Omega .
$$

We report in Table 1 the convergence history of our virtual element scheme (3.15). For each $\ell$ the Newton's method used up to 5 iterations with a tolerance Tol $=10^{-9}$. In particular, Table 1 summarizes the convergence history for the transverse displacement $u_{h}$ and for the Airy stress function $\psi_{h}$. As predicted by Theorem 4.2, an $\mathcal{O}(h)$ of convergence is clearly seen for $e_{2}\left(u_{h}\right)$ and $e_{2}\left(\psi_{h}\right)$. We also report $e_{0}\left(u_{h}\right), e_{1}\left(u_{h}\right), e_{0}\left(\psi_{h}\right)$ and $e_{1}\left(\psi_{h}\right)$, where an $\mathcal{O}\left(h^{2}\right)$ is observed. The exact and discrete solutions are depicted in Figure 3.

\subsection{Test 2}

In this test, we consider the canonical von Kármán equations ( $c f .(2.1))$ with non-homogeneous boundary conditions. We modify the right side of the second equation in (2.1) to compare the discrete solution with the continuous one, i.e.

$$
\left\{\begin{aligned}
\Delta^{2} u-[\psi, u] & =f & & \text { in } \Omega, \\
\Delta^{2} \psi+\frac{1}{2}[u, u] & =g & & \text { in } \Omega, \\
u=\partial_{\nu} u & =0 & & \text { on } \Gamma, \\
\psi & =\varphi_{0} & & \text { on } \Gamma, \\
\partial_{\nu} \psi & =\varphi_{1} & & \text { on } \Gamma .
\end{aligned}\right.
$$



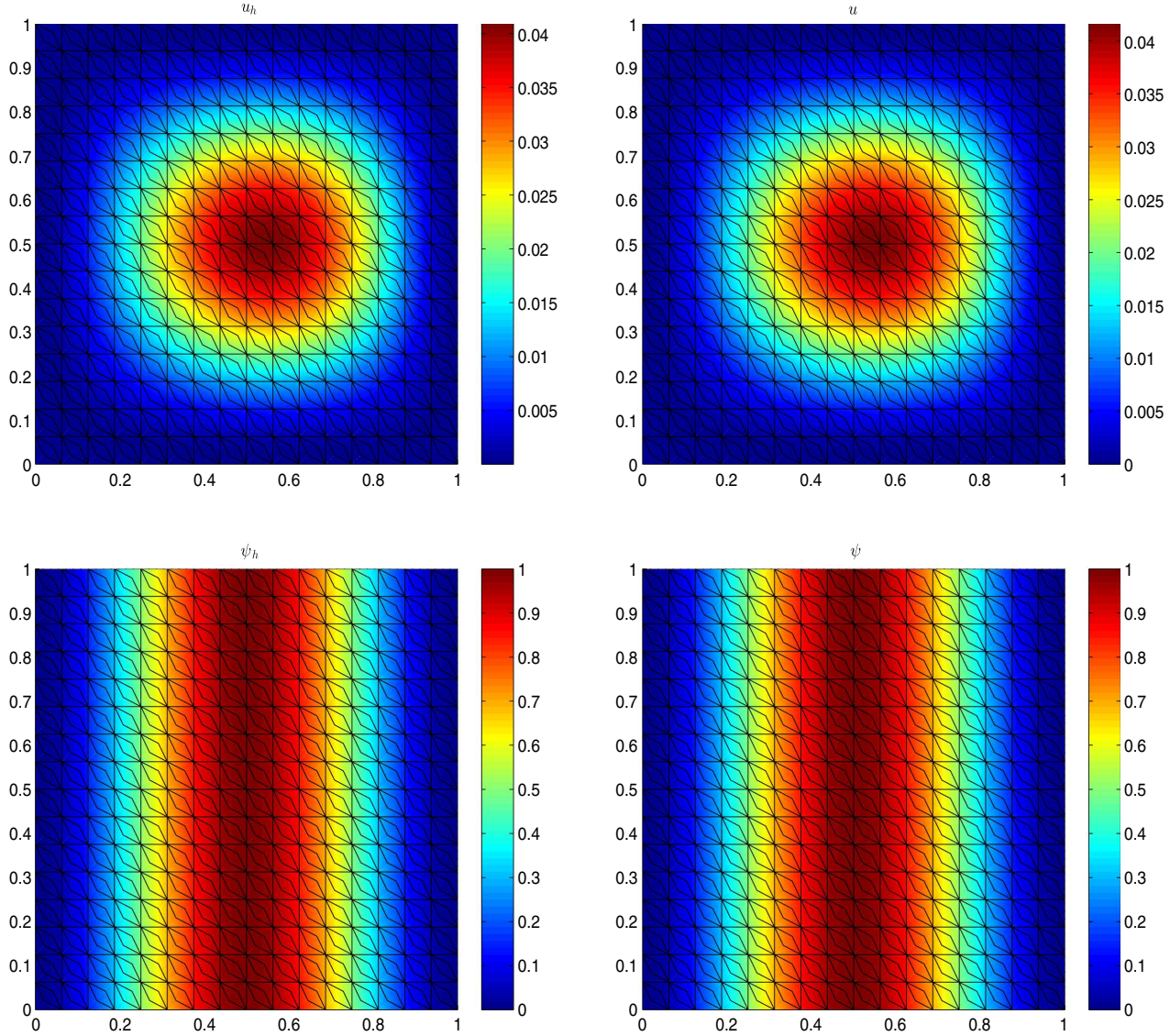

FiguRE 4. Test 2. $u_{h}$ (top left), $u$ (top right), $\psi_{h}$ (bottom left) and $\psi$ (bottom right).

Next, we consider the following lateral load forces $\varphi_{0}$ and $\varphi_{1}$

$$
\begin{aligned}
& \varphi_{0}(x, y)=\sin ^{2}(\pi x) \\
& \text { on } \Gamma \text {, } \\
& \varphi_{1}(x, y)=2 \pi \nu_{1}(x, y) \cos (\pi x) \sin (\pi x)
\end{aligned}
$$

and the right hand side functions $f$ and $g$ so that the exact solution of (5.2) is given by

$$
u(x, y)=x^{2} \sin (\pi y)^{2} \log (2 f-x)^{2} \quad \text { in } \Omega, \quad \text { and } \quad \psi(x, y)=\sin ^{2}(\pi x) \quad \text { in } \Omega .
$$

Table 2 reports the convergence history of our virtual element scheme (3.15) applied to solve system (5.2) on different polygonal meshes. Once again, for each $\ell$, the Newton's method used up to 5 iterations with a tolerance Tol $=10^{-9}$. In particular, Table 2 summarizes the convergence history for the transverse displacement $u_{h}$ and for the Airy stress function $\psi_{h}$. An $\mathcal{O}(h)$ of convergence is clearly seen for $e_{2}\left(u_{h}\right)$ and $e_{2}\left(\psi_{h}\right)$. We also report the errors $e_{0}\left(u_{h}\right), e_{1}\left(u_{h}\right), e_{0}\left(\psi_{h}\right)$ and $e_{1}\left(\psi_{h}\right)$, where an $\mathcal{O}\left(h^{2}\right)$ is observed.

In addition, in Figure 4 we display the discrete solution $\left(u_{h}, \psi_{h}\right)$ generated with the virtual scheme on a coarse mesh.

\subsection{Test 3}

In this test, we present a numerical example illustrating the performance of our virtual element scheme applied to the von Kármán system (2.4) with $f=0$ and different values of the parameter $\lambda$ ( $c f$. Rem. 2.1). Let 

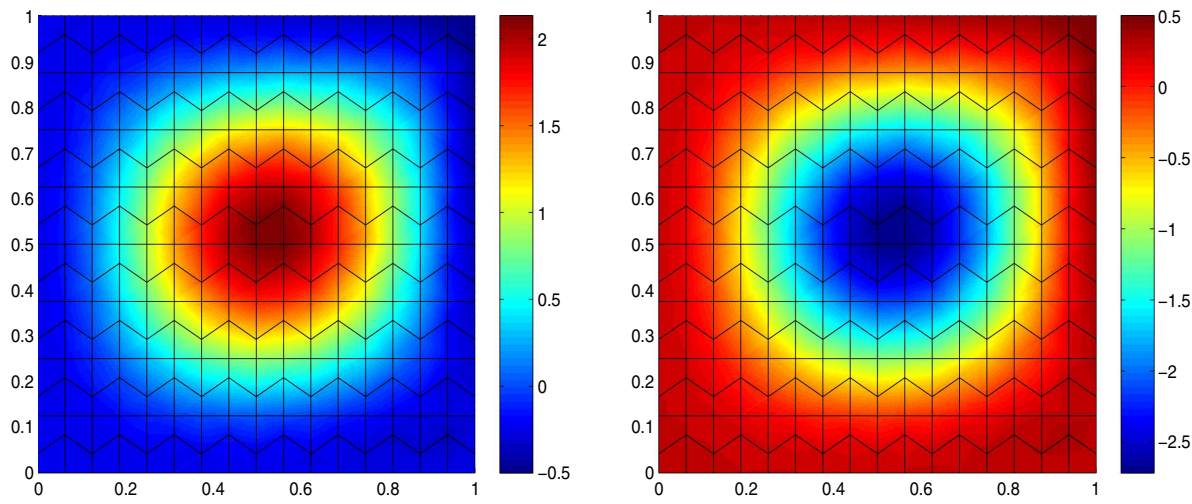

Figure 5. Test 3. $u_{1}:=u_{h}$ obtained with $\lambda=53, f=0$ and $\mathbf{u}^{0}(x, y)=\left(\frac{1}{4}\left(y x^{2}+1\right), \frac{1}{4}\left(y x^{2}+1\right)\right)$ (left). $u_{2}:=u_{h}$ obtained with $\lambda=53, f=0$ and $\mathbf{u}^{0}(x, y)=-\left(\frac{1}{4}\left(y x^{2}+1\right), \frac{1}{4}\left(y x^{2}+1\right)\right)($ right $)$.
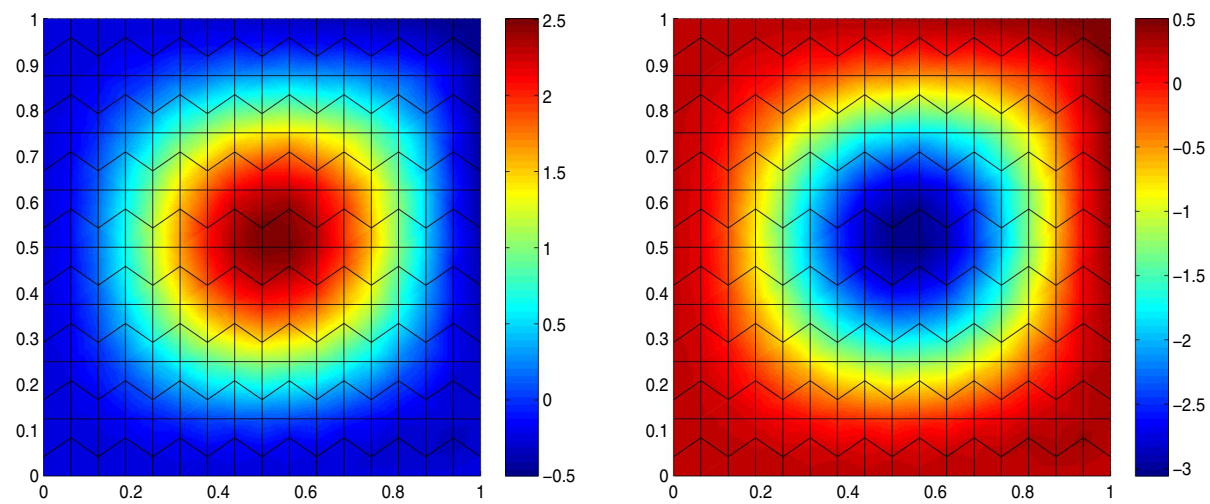

Figure 6 . Test 3. $u_{1}:=u_{h}$ obtained with $\lambda=55, f=0$ and $\mathbf{u}^{0}(x, y)=\left(\frac{1}{4}\left(y x^{2}+1\right), \frac{1}{4}\left(y x^{2}+1\right)\right)$ (left). $u_{2}:=u_{h}$ obtained with $\lambda=55, f=0$ and $\mathbf{u}^{0}(x, y)=-\left(\frac{1}{4}\left(y x^{2}+1\right), \frac{1}{4}\left(y x^{2}+1\right)\right)($ right $)$.
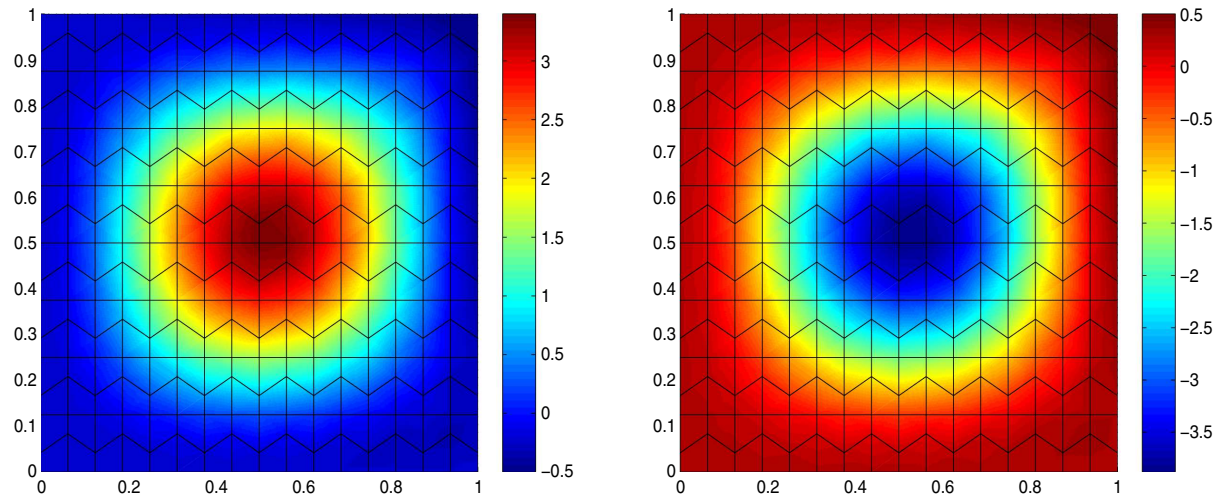

Figure 7. Test 3. $u_{1}:=u_{h}$ obtained with $\lambda=60, f=0$ and $\mathbf{u}^{0}(x, y)=\left(\frac{1}{4}\left(y x^{2}+1\right), \frac{1}{4}\left(y x^{2}+1\right)\right)$ (left). $u_{2}:=u_{h}$ obtained with $\lambda=60, f=0$ and $\mathbf{u}^{0}(x, y)=-\left(\frac{1}{4}\left(y x^{2}+1\right), \frac{1}{4}\left(y x^{2}+1\right)\right)($ right $)$. 
$\lambda^{\star}=52.34469 \ldots$ (see $\left.[17,43,44]\right)$ be the smallest eigenvalue $\lambda$ of the following buckling spectral problem $(c f$. $(2.5))$ :

$$
a^{\Delta}(u, v)=-\lambda a^{\nabla}(u, v) \quad \forall v \in H_{0}^{2}(\Omega),
$$

where the bilinear forms $a^{\Delta}(\cdot, \cdot)$ and $a^{\nabla}(\cdot, \cdot)$ have been defined in (2.8) and (2.9), respectively. In this particular case, as predicted by the theory in Theorem 5.9-2 of [31], there exist at least three solutions of problem (2.4) for $\lambda>\lambda^{\star}$ (see Rem. 2.1).

We have solved the discrete problem (3.15) using three values for $\lambda$. We take $\lambda=53, \lambda=55$ and $\lambda=60$. In addition, for each value of the parameter $\lambda$, we have used two different initial guess and trapezoidal meshes $\mathcal{T}_{h}^{1}$. On the left hand side of Figures 5-7, we illustrate the approximation of $u_{h}=: u_{1}$ obtained with the initial guess $\mathbf{u}_{h}^{0}(x, y)=\left(\frac{1}{4}\left(y x^{2}+1\right), \frac{1}{4}\left(y x^{2}+1\right)\right)$ and $\lambda=53,55$ and 60 , respectively. While, on the right hand side of Figures $5-$ 7 , we illustrate the approximation of $u_{h}=: u_{2}$ obtained with the initial guess $\mathbf{u}_{h}^{0}(x, y)=-\left(\frac{1}{4}\left(y x^{2}+1\right), \frac{1}{4}\left(y x^{2}+1\right)\right)$ and $\lambda=53,55$ and 60 , respectively. We can appreciate that $u_{1} \neq 0$ and $u_{2}=-u_{1}$, which confirm the existence of non zero solutions with opposite transverse displacement, as it was established in Theorem 5.9-2 of [31]. On the other hand, for $\mathbf{u}_{h}^{0} \equiv 0$, we obtained $\mathbf{u}_{h}=0$ for any value of $\lambda$, as it was established in Theorem 5.9-2 of [31].

Acknowledgements. The second author was partially supported by the National Agency for Research and Development, ANID-Chile, through FONDECYT project 1180913 and by project Centro de Modelamiento Matemático (AFB170001) of the PIA Program: Concurso Apoyo a Centros Científicos y Tecnológicos de Excelencia con Financiamiento Basal. The third author was partially supported by a ANID-Chile fellowship.

\section{REFERENCES}

[1] R.A. Adams and J.J. Fournier, Sobolev Spaces. Elsevier (2003).

[2] B. Ahmad, A. Alsaedi, F. Brezzi, L.D. Marini and A. Russo, Equivalent projectors for virtual element methods. Comput. Math. Appl. 66 (2013) 376-391.

[3] V. Anaya, M. Bendahmane, D. Mora and M. Sepúlveda, A virtual element method for a nonlocal FitzHugh-Nagumo model of cardiac electrophysiology. IMA J. Numer. Anal. 40 (2020) 1544-1576.

[4] P.F. Antonietti, L. Beirão da Veiga, D. Mora and M. Verani, A stream virtual element formulation of the Stokes problem on polygonal meshes. SIAM J. Numer. Anal. 52 (2014) 386-404.

[5] P.F. Antonietti, L. Beirão da Veiga, S. Scacchi and M. Verani, A $C^{1}$ virtual element method for the Cahn-Hilliard equation with polygonal meshes. SIAM J. Numer. Anal. 54 (2016) 34-56.

[6] E. Artioli, S. de Miranda, C. Lovadina and L. Patruno, A family of virtual element methods for plane elasticity problems based on the Hellinger-Reissner principle. Comput. Methods Appl. Mech. Eng. 340 (2018) 978-999.

[7] K. Atkinson and W. Han, Theoretical Numerical Analysis. Texts in Applied Mathematics. Springer, Dordrecht (2009).

[8] L. Beirão da Veiga, F. Brezzi, A. Cangiani, G. Manzini, L.D. Marini and A. Russo, Basic principles of virtual element methods. Math. Models Methods Appl. Sci. 23 (2013) 199-214.

[9] L. Beirão da Veiga, F. Brezzi and L.D. Marini, Virtual elements for linear elasticity problems. SIAM J. Numer. Anal. 51 (2013) 794-812.

[10] L. Beirão da Veiga, F. Brezzi, L.D. Marini and A. Russo, The hitchhiker's guide to the virtual element method. Math. Models Methods Appl. Sci. 24 (2014) 1541-1573.

[11] L. Beirão da Veiga, C. Lovadina and D. Mora, A virtual element method for elastic and inelastic problems on polytope meshes. Comput. Methods Appl. Mech. Eng. 295 (2015) 327-346.

[12] L. Beirão da Veiga, F. Brezzi, F. Dassi, L.D. Marini and A. Russo, Virtual element approximation of 2D magnetostatic problems. Comput. Methods Appl. Mech. Eng. 327 (2017) 173-195.

[13] L. Beirão da Veiga, C. Lovadina and A. Russo, Stability analysis for the virtual element method. Math. Models Methods Appl. Sci. 27 (2017) 2557-2594.

[14] L. Beirão da Veiga, F. Brezzi, F. Dassi, L.D. Marini and A. Russo, Lowest order virtual element approximation of magnetostatic problems. Comput. Methods Appl. Mech. Engrg. 332 (2018) 343-362.

[15] L. Beirão da Veiga, C. Lovadina and G. Vacca, Virtual elements for the Navier-Stokes problem on polygonal meshes. SIAM J. Numer. Anal. 56 (2018) 1210-1242.

[16] L. Beirão da Veiga, D. Mora and G. Rivera, Virtual elements for a shear-deflection formulation of Reissner-Mindlin plates. Math. Comput. 88 (2019) 149-178.

[17] P.E. Bjørstad and B.P. Tjøstheim, High precision solutions of two fourth order eigenvalue problems. Computing 63 (1999) 97-107.

[18] H. Blum and R. Rannacher, On the boundary value problem of the biharmonic operator on domains with angular corners. Math. Methods Appl. Sci. 2 (1980) 556-581. 
[19] S.C. Brenner, Q. Guan and L.-Y. Sung, Some estimates for virtual element methods. Comput. Methods Appl. Math. 17 (2017) $553-574$.

[20] S.C. Brenner, M. Neilan, A. Reiser and L.-Y. Sung, A $C^{0}$ interior penalty method for a von Kármán plate. Numer. Math. 135 (2017) 803-832.

[21] S.C. Brenner and L.R. Scott, The Mathematical Theory of Finite Element Methods. Springer, New York (2008).

[22] H. Brezis, Functional Analysis, Sobolev Spaces and Partial Differential Equations. Springer, New York (2011).

[23] F. Brezzi, Finite element approximations of the von Kármán equations. RAIRO Anal. Numér. 12 (1978) 303-312.

[24] F. Brezzi and L.D. Marini, Virtual element methods for plate bending problems. Comput. Methods Appl. Mech. Eng. 253 (2013) 455-462.

[25] E. Cáceres and G.N. Gatica, A mixed virtual element method for the pseudostress-velocity formulation of the Stokes problem. IMA J. Numer. Anal. 37 (2017) 296-331.

[26] A. Cangiani, P. Chatzipantelidis, G. Diwan and E.H. Georgoulis, Virtual element method for quasilinear elliptic problems. IMA J. Numer. Anal. 40 (2020) 2450-2472.

[27] A. Cangiani, G. Manzini and O.J. Sutton, Conforming and nonconforming virtual element methods for elliptic problems. IMA J. Numer. Anal. 37 (2017) 1317-1354.

[28] C. Carstensen, G. Mallik and N. Nataraj, A priori and a posteriori error control of discontinuous galerkin finite element methods for the von kármán equations. IMA J. Numer. Anal. 39 (2018) 167-200.

[29] C. Chinosi, Virtual elements for the Reissner-Mindlin plate problem. Numer. Methods Part. Differ. Equ. 34 (2018) $1117-1144$.

[30] C. Chinosi and L.D. Marini, Virtual element method for fourth order problems: $L^{2}$-estimates. Comput. Math. Appl. 72 (2016) $1959-1967$.

[31] P.G. Ciarlet, Mathematical Elasticity. Vol. II. Theory of Plates. North-Holland, Amsterdam (1997).

[32] P.G. Ciarlet, The Finite Element Method for Elliptic Problems. SIAM, Philadelphia (2002).

[33] P.G. Ciarlet and P. Rabier, Les équations de von Kármán. In: Vol. 826 of Lecture Notes in Mathematics. Springer, Berlin (1980).

[34] A. Ern and G.L. Guermond, Theory and practice of finite elements. In: Vol. 159 of Applied Mathematical Sciences. SpringerVerlag, New York (2004).

[35] G.N. Gatica, M. Munar and F.A. Sequeira, A mixed virtual element method for the Navier-Stokes equations. Math. Models Methods Appl. Sci. 28 (2018) 2719-2762.

[36] G.N. Gatica, M. Munar and F.A. Sequeira, A mixed virtual element method for the Boussinesq problem on polygonal mesh. To appear in: J. Comput. Math. DOI: 10.4208/jcm.2001-m2019-0187

[37] F. Gazzola and Y. Wang, Modeling suspension bridges through the von Kármán quasilinear plate equations. Contrib. Nonlinear Elliptic Equ. Syst. 86 (2015) 269-297.

[38] G.H. Knightly, An existence theorem for the von Kármán equations. Arch. Ration. Mech. Anal. 27 (1967) $233-242$.

[39] G. Mallik and N. Nataraj, Conforming and nonconforming finite element methods for canonical von Kármán equations. Int. J. Adv. Eng. Sci. Appl. Math. 7 (2015) 86-95.

[40] G. Mallik and N. Nataraj, Conforming finite element methods for the von Kármán equations. Adv. Comput. Math. 42 (2016) 1031-1054.

[41] G. Mallik and N. Nataraj, A nonconforming finite element approximation for the von Karman equations. ESAIM:M2AN 50 (2016) 433-454.

[42] L. Mascotto, I. Perugia and A. Pichler, Non-conforming harmonic virtual element method: $h$ - and $p$-versions. J. Sci. Comput. 77 (2018) 1874-1908.

[43] D. Mora and R. Rodríguez, A piecewise linear finite element method for the buckling and the vibration problems of thin plates. Math. Comput. 78 (2009) 1891-1917.

[44] D. Mora and I. Velásquez, Virtual element for the buckling problem of Kirchhoff-Love plates. Comput. Methods Appl. Mech. Eng. 360 (2020) 112687.

[45] D. Mora, G. Rivera and I. Velásquez, A virtual element method for the vibration problem of Kirchhoff plates. ESAIM:M2AN 52 (2018) 1437-1456.

[46] I. Perugia, P. Pietra and A. Russo, A plane wave virtual element method for the Helmholtz problem. ESAIM:M2AN 50 (2016) 783-808.

[47] G. Vacca, An $H^{1}$-conforming virtual element for Darcy and Brinkman equations. Math. Models Methods Appl. Sci. 28 (2018) 194.

[48] T. Von Kármán, Festigkeitsprobleme im maschinenbau. Encycl. der Mathematischen Wissenschaften, Leipzig IV/4 (1910) 348-352.

[49] Y. Wang, An evolution von Kármán equation modeling suspension bridges. Nonlinear Anal. 169 (2018) 59-78.

[50] P. Wriggers, W.T. Rust and B.D. Reddy, A virtual element method for contact. Comput. Mech. 58 (2016) $1039-1050$. 